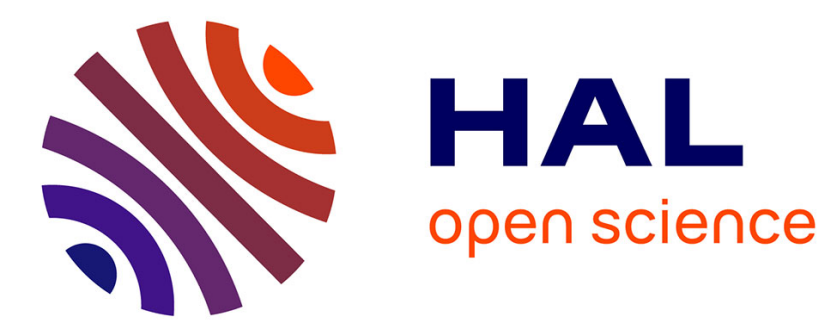

\title{
Aerodynamic Optimization Using a Parallel Asynchronous Evolutionary Algorithm Controlled by Strongly Interacting Demes
}

Kyriakos C. Giannakoglou, Varvara G Asouti

\section{- To cite this version:}

Kyriakos C. Giannakoglou, Varvara G Asouti. Aerodynamic Optimization Using a Parallel Asynchronous Evolutionary Algorithm Controlled by Strongly Interacting Demes. Engineering Optimization, 2009, 41 (03), pp.241-257. 10.1080/03052150802415665 . hal-00514317

\section{HAL Id: hal-00514317 \\ https://hal.science/hal-00514317}

Submitted on 2 Sep 2010

HAL is a multi-disciplinary open access archive for the deposit and dissemination of scientific research documents, whether they are published or not. The documents may come from teaching and research institutions in France or abroad, or from public or private research centers.
L'archive ouverte pluridisciplinaire HAL, est destinée au dépôt et à la diffusion de documents scientifiques de niveau recherche, publiés ou non, émanant des établissements d'enseignement et de recherche français ou étrangers, des laboratoires publics ou privés. 


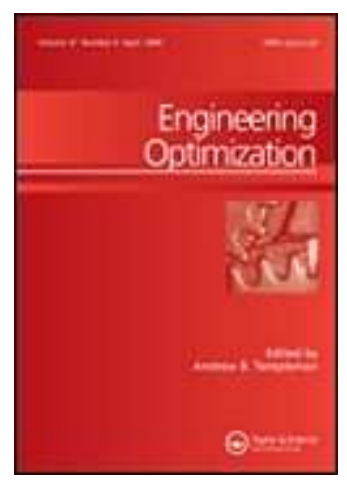

\section{Aerodynamic Optimization Using a Parallel Asynchronous Evolutionary Algorithm Controlled by Strongly Interacting Demes}

\begin{tabular}{|c|c|}
\hline Journal: & Engineering Optimization \\
\hline Manuscript ID: & GENO-2008-0091.R2 \\
\hline Manuscript Type: & Original Article \\
\hline $\begin{array}{r}\text { Date Submitted by the } \\
\text { Author: }\end{array}$ & 14-Aug-2008 \\
\hline Complete List of Authors: & $\begin{array}{l}\text { Giannakoglou, Kyriakos; National Technical University of Athens } \\
\text { Asouti, Varvara; National Technical University of Athens }\end{array}$ \\
\hline Keywords: & $\begin{array}{l}\text { Asynchronous Evolutionary Algorithm, Parallelization, Aerodynamic } \\
\text { Shape Optimization, Multi-objective Optimization }\end{array}$ \\
\hline \multicolumn{2}{|c|}{$\begin{array}{l}\text { Note: The following files were submitted by the author for peer review, but cannot be converted } \\
\text { to PDF. You must view these files (e.g. movies) online. }\end{array}$} \\
\hline $\begin{array}{l}\text { Asouti.tex } \\
\text { bbold11.tfm } \\
\text { gENO2e.cls } \\
\text { natbib.cfg } \\
\text { refs.tex } \\
\text { symbols.tex }\end{array}$ & \\
\hline
\end{tabular}

\section{S ScholaroNE" \\ Manuscript Central}




\title{
Aerodynamic Optimization Using a Parallel Asynchronous Evolutionary Algorithm Controlled by Strongly Interacting Demes
}

\author{
Varvara G. Asouti and Kyriakos C. Giannakoglou* \\ National Technical University of Athens, School of Mechanical Engineering, \\ Lab. of Thermal Turbomachines, Parallel CFD \& Optimization Unit, \\ P.O. Box 64069, Athens 157 10, Greece
}

(July 2008)

\begin{abstract}
A parallel asynchronous evolutionary algorithm controlled by strongly interacting demes for single- and multi-objective optimization problems is proposed. It is suitable for, even nonhomogeneous, multiprocessor systems, ensuring maximum exploitation of the available processors. The search algorithm utilizes a structured topology of evaluation agents organized in a number of inter-communicating demes arranged on a 2D supporting mesh. Once an evaluation terminates and a processor becomes idle, a series of intra- and inter-deme processes determine the next agent to undergo evaluation on this specific processor. Real coding and differential evolution operators are used. Mathematical and aerodynamic-turbomachinery optimization problems are presented to assess the proposed method in terms of CPU cost, parallel efficiency and quality of solutions obtained within a predefined number of evaluations. Comparisons with conventional evolutionary algorithms, parallelized based on the master-slave model on the same computational platform, are presented.
\end{abstract}

Keywords: Asynchronous Evolutionary Algorithm; Parallelization; Aerodynamic Shape Optimization; Multi-objective Optimization.

\section{Introduction}

Nowadays Evolutionary Algorithms (EAs) are broadly used in engineering optimization. They are global search tools which may accommodate any in-house or commercial evaluation software, to solve multi-objective or multi-disciplinary problems by approximating the Pareto front of optimal solutions. An EA-based optimization requires an excessive amount of calls to the evaluation software which increases the CPU cost, especially in case of computationally demanding evaluation tools. To overcome this weakness, our group has proposed different strategies, including the use of surrogate evaluation models (Metamodel Assisted EAs-MAEAs), distributed EAs (DEAs) and hierarchical algorithms (HEAs) (Giannakoglou 2002, Kampolis et al. 2007, Kampolis and Giannakoglou 2008, Karakasis and Giannakoglou 2006), to be used separately or in combination. All the aforementioned papers refer to synchronous EAs, where the use of multiprocessing is solely restricted to the concurrent evaluation of candidate solutions, without altering the convergence of the corresponding sequential algorithms. To reduce further the wall clock optimization time, Parallel EAs (PEAs), based on the partitioning of search in simultaneously processed subproblems, have been proposed in the literature. PEAs (Alba and Troya 2001, Alba et al. 2002, Lim et al. 2007, Melab et al. 2006) are perfectly suited for cluster or grid computing. Parallel search methods have

*Corresponding author. Email: kgianna@central.ntua.gr 
also been presented by the authors' group (Giannakoglou et al. 2007, Liakopoulos et al. 2008). In contrast to the presently proposed asynchronous EA, the last two publications rely on generation-based EAs with a shift of emphasis from cluster to grid computing. In the literature, various classifications of PEAs can be found (Alba and Tomassini 2002, Cantú-Paz 1998, Nowostawski and Poli 1999).

Depending on the population distribution, PEAs can be classified into singleand multi-population ones. Multi-population PEAs can be further classified into distributed and cellular EAs, according to the topology of sub-populations. The parallelization of single-population EAs is typically based on the master-slave paradigm and their parallel efficiency is determined by the number of processors, the population size, the parallelization or not of the evaluation software, etc. Synchronous PEAs, where the master has to wait all slaves to complete their evaluations before proceeding to the next generation, cannot attain $100 \%$ parallel efficiency, since some CPUs remain idle for a while due to the synchronization barrier. A way to theoretically overcome this problem is by defining as many offspring as the number of the available processors or a multiple of them. However, even if the number of processors is fixed and the parallel system is homogeneous, this is not a real remedy to the problem as there is no guarantee that all of the evaluations have the same CPU cost. For instance, in the EA-based design of an aerodynamic body using a CFD code as evaluation software, some of the emerging candidate shapes may cause complex flow features which need more iterations and, thus, more CPU time to converge. So, the CPU cost per evaluation often varies. In contrast, asynchronous master-slave PEAs may safely overcome this problem.

In view the above, the scope of this paper is to overcome limitations of synchronous EAs by proposing an asynchronous EA controlled by strongly interacting (overlapping) demes for multiprocessor platforms, which may optimally use any number of available processors, in either single- or multi-objective problems. The proposed method is new with just a few similarities with the so-called cellular EAs (cEAs), (Manderick and Spiessens 1989, Muhammad et al. 1997). It is based on a spatially distributed population, arranged in demes with six nodes each. A deme overlaps with its four neighbors. This justifies the term "strongly interacting demes" since the majority of search nodes (four out of five per deme; see Section 2 ) are shared. The evolutionary operators apply within each deme only. The most important organizational features, such as the arrangement of search agents on the nodes of a periodic 2D supporting mesh, their clustering into overlapping demes and the use of age- and cost-based criteria for the selection of the next individual to be evaluated, are analyzed in detail. The asynchronous operation ensures that all available CPUs remain fully busy during the optimization.

The proposed method is demonstrated firstly on mathematical functions and, then, on aerodynamic (including turbomachinery) design problems. Computations were performed on the Parallel CFD \& Optimization Unit's multiprocessor platform which consists of 3 clusters. The first has 22 nodes (35 cores - P4, 32bit, 35GB total RAM), the second has 32 nodes (80 cores - P4 \& Xeon, 64 bit, 105GB total RAM) and the third is based on blade servers (ten 64 bit, $2 \times$ quad core Xeon nodes, i.e. 80 cores) and a total RAM of $80 \mathrm{~GB}$. In the applications section, CPU stands for each core of the aforementioned clusters. 


\section{The Proposed Parallel Asynchronous Evolutionary Algorithm}

\subsection{Fundamentals}

A distinguishing feature of the proposed algorithm is the topological structure of search or evaluation agents and supporting poles, as defined below. Both are basic elements of the proposed algorithm, lying on the nodes of a $2 \mathrm{D}$ supporting structured mesh which is considered to be periodic across both pairs of opposite sides. Periodicity implies that the left-most mesh nodes are virtually linked to the right-most ones and so do the nodes lying along its upper and lower rows. The mesh size $n_{1} \times n_{2}$ ( $n_{1}$ and $n_{2}$ must be even for periodicity to apply) is user defined by considering the problem complexity and the number of available processors. The mesh is partitioned into demes as shown in Figure 1. Each deme (denoted by $\mathcal{D}_{p}$ ) consists of six mesh nodes, namely a pole and five evaluation agents and overlaps with four adjacent demes. This overlapping is such that, a deme shares four out of its five agents with the four adjacent demes; there is only one nonshared agent in each deme. On a $n_{1} \times n_{2}$ mesh, the number of poles is equal to $N_{\text {poles }}=\frac{N_{\text {mesh }}}{4}$ and the number of evaluation agents is equal to $N_{\text {agents }}=\frac{3 N_{\text {mesh }}}{4}$, where $N_{\text {mesh }}=n_{1} n_{2}$. Let us consider that $N_{\text {agents }} \geq N_{C P U}$, where $N_{C P U}$ is the total number of processors which are available during the optimization. Shared and nonshared agents may undergo evaluations. Poles are considered to be the front-ends of demes and are used to store the best individual computed by their agents thus far. They are assigned a dynamically changing priority (priorities may indifferently be associated with a deme or the corresponding pole) that helps determining the next deme and, through that, the next agent to undergo evaluation, as it will be discussed below.

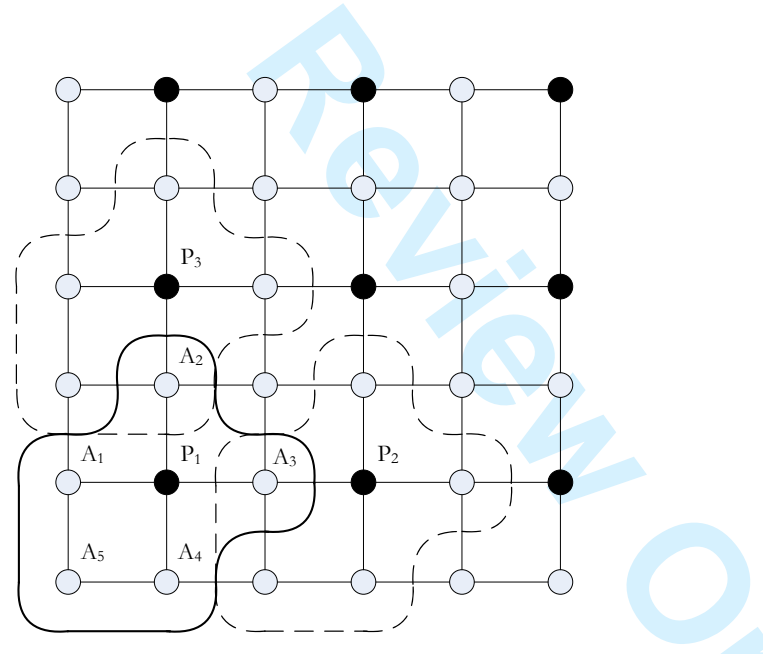

Figure 1. Topology of a $6 \times 6$ supporting mesh; three of its demes are clearly marked. All poles are marked with black circles. For the deme represented by pole $P_{1}$, agent $A_{3}$ is search-agent shared between demes centered at $P_{1}$ and $P_{2}$, whereas agent $A_{5}$ is the only non-shared one.

At the beginning of optimization, each processor starts running an evaluation associated with an agent selected at random. If $N_{\text {agents }}>N_{C P U}$, some of the agents remain inactive. The next evaluation request will be forwarded to the first processor that becomes available (idle) after completing the previously undertaken evaluation. The new request will be sent by any of the agents of the deme with the highest priority, as decided after updating all deme priorities, by considering their age and current cost value. Before starting a new evaluation, a new candidate solution is formed via intra- and inter-deme exchange of information and evolution operators. Hereafter, we focus on multi-objective algorithms, from which their 
single-objective counterpart can readily be extracted. Assume that $M$ functions must be minimized,

$$
\min \vec{f}(\vec{x})=\min \left\{f_{1}(\vec{x}), \ldots, f_{M}(\vec{x})\right\}
$$

where the design variables' vector $\vec{x}$ belongs to the subset of $R^{N}$ defined by their known lower and upper bounds. Constraints, if any, are taken into account by equally penalizing all the $f_{m}$ values of infeasible individuals. For $M_{c}$ inequality constraints cast in the form

$$
c_{j}(\vec{x}) \leq c_{j}^{\text {thres }}, \quad j=1, \ldots, M_{c}
$$

the exponential penalty term is defined by

$$
P_{j}=\left\{\begin{array}{cc}
1 & \text { if } c_{j}(\vec{x}) \leq c_{j}^{\text {thres }} \\
\exp \left\{\alpha \frac{c_{j}-c_{j}^{\text {thres }}}{c_{j}^{\text {dead }}-c_{j}^{\text {thres }}}\right\} & \text { if } \\
\infty & c_{j}^{\text {thres }}<c_{j}(\vec{x})<c_{j}^{\text {dead }} \\
\infty & \text { if } c_{j}(\vec{x}) \geq c_{j}^{\text {dead }}
\end{array}\right.
$$

where $\alpha$ is a user-defined positive constant and $c_{j}^{\text {dead }}>c_{j}^{\text {thres }}$ is a second userdefined threshold value for absolutely unacceptable ("unacceptably infeasible") emerging solutions. $P_{j}$ multiplies all $f_{m}$ values computed by the evaluation software. Hereafter, $\vec{f}(\vec{x})$ or $f_{m}(\vec{x})$ denote penalized costs (i.e. $f_{m}(\vec{x})=f_{m}(\vec{x}) \prod_{j=1}^{M_{c}} P_{j}$ ) which coincide with the real costs of feasible solutions only.

For $M>1$, dominance criteria are used to compare two solutions $\vec{x}_{1}$ and $\vec{x}_{2}$. If $\vec{x}_{1}$ is no worse than $\vec{x}_{2}$ for all objectives $f_{m}$ and wholly better for at least one objective, then $\vec{x}_{1}$ dominates $\vec{x}_{2}$ and this is denoted by $\vec{x}_{1} \succ \vec{x}_{2}$. In this case $f_{m}\left(\vec{x}_{1}\right) \leq f_{m}\left(\vec{x}_{2}\right)$ and $f_{m}\left(\vec{x}_{1}\right)<f_{m}\left(\vec{x}_{2}\right)$ for, at least, one $m \in[1, M]$. In addition, $\vec{x}_{1} \nsucc \vec{x}_{2}$ (or $\vec{x}_{2} \nsucc \vec{x}_{1}$ ) denotes two non-dominating individuals, where neither of them dominates the other. During the evolution, the non-dominating among the already evaluated individuals are recorded in the continuously updated archival set $\mathcal{P}_{a}$ which progressively approaches the Pareto front of optimal solutions.

\subsection{Pole Displacement}

As soon as an evaluation of $\vec{x}_{a g e n t}$ is over, a decision should be made on whether $\vec{x}_{\text {agent }}$ must displace the corresponding pole (for non-shared agents) or poles (for shared agents). This decision is based on the comparison of $\vec{x}_{\text {agent }}$ and the solutions currently stored at the corresponding pole(s) $\vec{x}_{\text {pole }}$. If $\vec{x}_{\text {agent }} \succ \vec{x}_{\text {pole }}, \vec{x}_{\text {pole }}$ is displaced by $\vec{x}_{\text {agent }}$. If $\vec{x}_{\text {pole }} \succ \vec{x}_{\text {agent }}$, no displacement takes place. In all other cases (no absolute domination, $\vec{x}_{\text {agent }} \nsucc \vec{x}_{\text {pole }}$ ), other criteria including a partially stochastic selection, are used. These criteria primarily depend on whether $\vec{x}_{\text {agent }}$ and $\vec{x}_{\text {pole }}$ belong to the $\mathcal{P}_{a}$ set or not. In particular, $\vec{x}_{\text {agent }}$ displaces $\vec{x}_{\text {pole }}$ if $\vec{x}_{\text {agent }} \in \mathcal{P}_{a} \& \vec{x}_{\text {pole }} \notin \mathcal{P}_{a}$. Also, $\vec{x}_{\text {agent }}$ may displace $\vec{x}_{\text {pole }}$ with $50 \%$ probability if $\vec{x}_{\text {agent }} \in \mathcal{P}_{a} \& \vec{x}_{\text {pole }} \in \mathcal{P}_{a}$ or $\vec{x}_{\text {agent }} \notin \mathcal{P}_{a} \& \vec{x}_{\text {pole }} \notin \mathcal{P}_{a}$. In all other cases, $\vec{x}_{\text {pole }}$ remains intact.

\subsection{Selection of the Next Agent to Undergo Evaluation}

An inter-deme process determines the next agent to undergo evaluation on the currently idle processor. This decision depends on priority values given to poles. The priority $\operatorname{Pr}_{p}$ of pole $p$ is the product of the age-based $P r_{p}^{a g e}$ and cost-based 
$\operatorname{Pr}_{p}^{\text {cost }}$ priorities, namely

$$
\operatorname{Pr}_{p}=\operatorname{Pr}_{p}^{a g e} \operatorname{Pr}_{p}^{\text {cost }}
$$

The age $A_{k}$ of agent $k$ is defined as the difference of the serial number of the last evaluation carried out by this agent from the serial number of the current evaluation. Pole $p$ takes on the average age of its agents,

$$
\bar{A}_{p}=\frac{1}{5} \sum_{k \in \mathcal{D}_{p}} A_{k}
$$

Based on the ages $\bar{A}_{p}$ of all poles, age-based priority of poles are defined by

$$
\operatorname{Pr}_{p}^{a g e}=\frac{\bar{A}_{p}}{\max \left(\bar{A}_{1}, \ldots, \bar{A}_{N_{\text {poles }}}\right)}
$$

The cost-based priority function is defined using strength- and density-based criteria applied to the individuals stored at poles. Its definition was "inspired" by the SPEA algorithm (Zitzler et al. 2002). In the objective function space, the strength $S_{p}$ of each pole is equal to the number of poles this pole dominates. By definition, all $S_{p}$ take on integer values in $\left[0, N_{\text {poles }}-1\right]$. A raw fitness value $R_{p}$ is, then, given to each pole $p$ by summing up the strengths of poles $p^{*}$ dominating $p$, namely

$$
R_{p}=\sum_{p^{*} \succ p} S_{p^{*}}
$$

Also, at each pole, a density value $D_{p}$ is defined as

$$
D_{p}=\frac{1}{d_{p}+2}
$$

where $d_{p}$ is equal to its distance to the $k$-th nearest pole defined as in (Zitzler et al. 2002). Then, the cost-based priority value for each pole is given by

$$
\operatorname{Pr}_{p}^{\text {cost }}=\frac{\Phi_{p}}{\Phi_{\max }-\Phi_{\min }}, \Phi_{p}=D_{p}+R_{p}
$$

where $\Phi_{\max }=\max \left\{\Phi_{1}, \ldots, \Phi_{N_{\text {poles }}}\right\}$ and $\Phi_{\min }=\min \left\{\Phi_{1}, \ldots, \Phi_{N_{\text {poles }}}\right\}$

The next agent to be evaluated belongs to the deme with the maximum priority $P r_{p}$, as computed by Eq. 4. According to a simple intra-deme process, among the five agents of this deme, the one with the maximum age is selected to undergo evaluation.

\subsection{Recombination and Mutation}

The recombination scheme is similar to the one used in differential evolution algorithm (Storn and Price 1997) and applies within the previously selected deme. The new member to be evaluated is formed by superimposing the weighted difference between two agents of the same deme to the individual currently stored at the pole 
$\vec{x}_{\text {pole }}$ as follows

$$
\vec{x}_{k}=\vec{x}_{\text {pole }}+\omega\left(\vec{x}_{k_{1}}-\vec{x}_{k_{2}}\right), \quad k_{1}, k_{2} \in S_{p} \& k_{1} \neq k_{2}
$$

where $\omega$ is a random number between 0 and 1 .

A non-uniform mutation scheme is applied with a small user-defined probability. This scheme is similar to the one presented in (Michalewicz 1994), with zero exponent so as to make mutation independent of the generation counter which does not exist in an asynchronous method. In case the new member's value for any design variable $i$ falls outside the pre-defined bounds $\left[b_{l o w}^{i}, b_{u p}^{i}\right]$, a correction scheme is applied, as follows

$$
\begin{aligned}
& x_{k}^{i}=b_{\text {low }}^{i}+\left|x_{k}^{i}-b_{\text {low }}^{i}\right|, \text { if } x_{k}^{i}<b_{\text {low }}^{i} \\
& x_{k}^{i}=b_{u p}^{i}-\left|x_{k}^{i}-b_{u p}^{i}\right|, \text { if } x_{k}^{i}>b_{u p}^{i}
\end{aligned}
$$

The proposed algorithm in pseudocode is given in Algorithm 1.

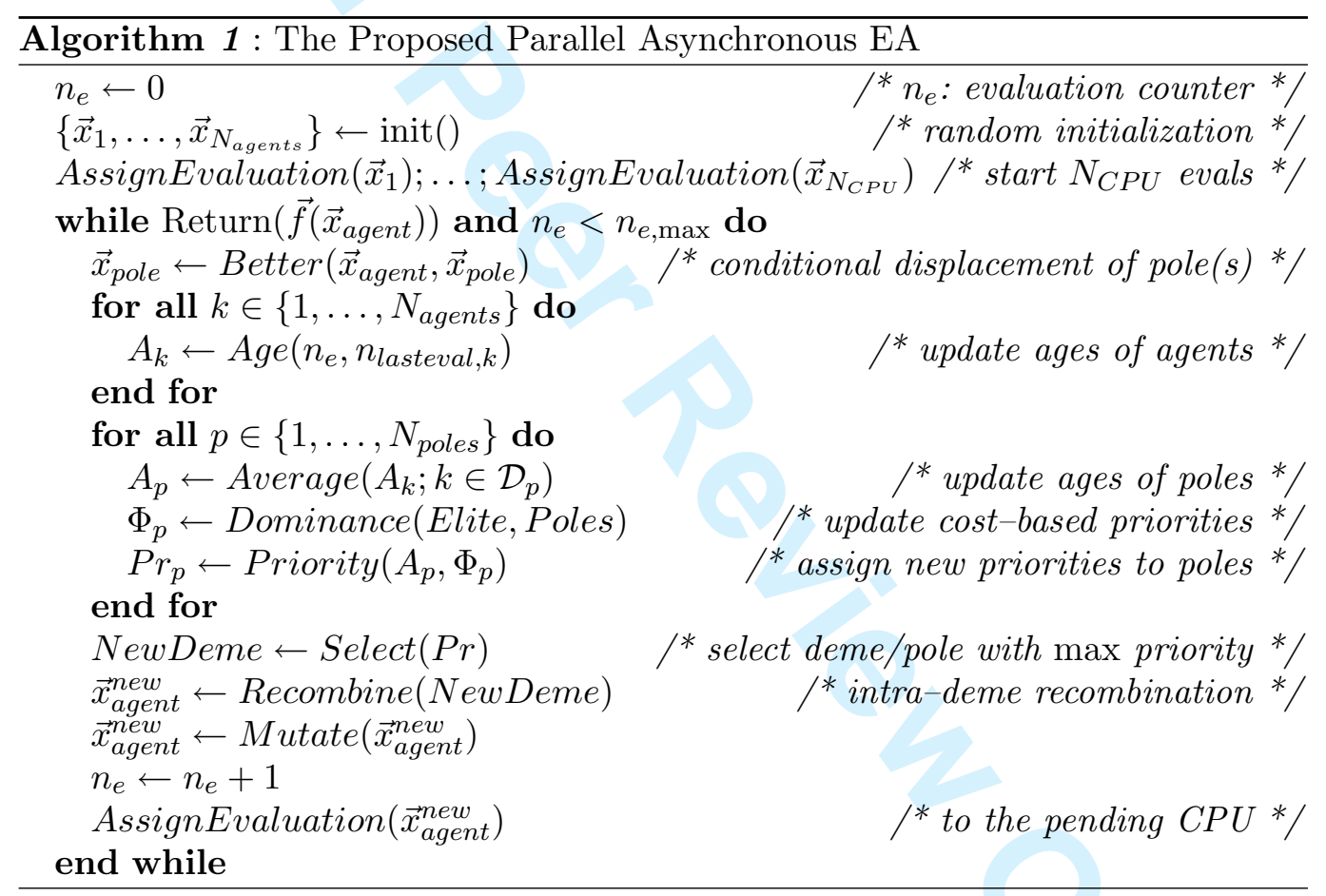

\section{Method Application \& Assessment}

The proposed method (hereafter abbreviated to Asynchronous EA or AEA) is tested on constrained or unconstrained, single- and two-objective mathematical minimization problems before proceeding to more demanding design problems in turbomachinery and external aerodynamics. The following cases aim to (a) show the superiority of the AEA compared to conventional EAs, (b) understand the action of strongly interacting demes, (c) assess the importance of using prioritycontrolled schemes, (d) demonstrate the excellent parallel efficiency of the AEA by practically eliminating the idle CPU time, (e) study the role of the supporting mesh size. 


\section{Page 7 of 50 Engineering Optimization Asouti \\ Engineering Optimization}

Engineering Optimization

\subsection{Mathematical Benchmarks}

The first mathematical benchmark is concerned with the minimization of the shifted sphere function, $f(\vec{x})=\sum_{i=1}^{N} z_{i}^{2}-450, \vec{z}=\vec{x}-\vec{o} \in R^{N}$, with $N=10$, 30 and 50 design variables. This unimodal and separable function is one of the CEC'05 (Suganthan et al. 2005) test functions. According to the CEC'05 criteria, 25 independent runs with up to $10000 \times N$ evaluations each were carried out on 25 CPUs (second cluster) and a $10 \times 10$ supporting mesh. The runs were performed using different seed states of the pseudo-random number generator (RNG). Note that, in contrast to synchronous EAs, the same RNG leads to different convergence each time the AEA run is repeated, due to the asynchronous operation of the method and possible changes in CPU loading. The mean convergence histories for the three $N$ values are plotted in Figure 2 and statistical results can be found in Table 1. The results (presented in terms of error values, i.e. differences between the computed function values and the exact solution $\vec{o}$ ) are very good compared to those presented in the CEC'05 Special Session on Real-Parameter Optimization (http://www3.ntu.edu.sg/home/EPNSugan/).

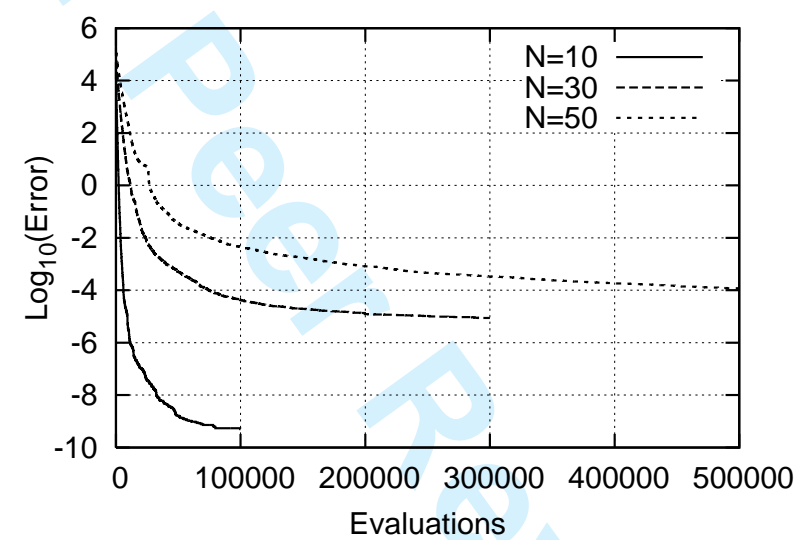

Figure 2. Shifted Sphere. Mean convergence history of the proposed AEA for $N=10,30$ and 50 design variables.

The next case is on the minimization of the Ackley's function (Ackley 1987), in its generalized form presented by Bäck (Bäck 1996), with $N=30$ design variables. This multimodal (with several local minima), non-separable function is used to compare the proposed AEA with a conventional EA and study the role of the priority function in AEAs. A $10 \times 10$ supporting mesh $\left(N_{\text {agents }}=75, N_{\text {poles }}=25\right)$ and $20 \mathrm{CPUs}$ (of the first and second cluster) were used. The optimal solutions achieved after 30 runs (stopping criterion: 10000 evaluations) have $f_{\text {mean }}=0.8963$ and standard deviation $s=0.3861$ while the conventional EA yields $f_{\text {mean }}=1.8732$ and $s=0.5366$. The mean convergence history of the 30 runs is plotted in Figure 3 (left). To compare the mean values of the AEA and the synchronous and generation-based EA, a t-test (which yields to $t_{0}=8.0925$ ) ensures that the proposed method is significantly better than the conventional EA.

A study on the use of the priority-based criterion, Eqs. 4, 6 and 9, in order to define the next agent to undergo evaluation, follows. For the purpose of comparison, instead of the aforementioned criterion, a random selection of the next inactive agent to undergo evaluation was used and the 30 runs were repeated. The comparison of results with and without priority control are presented in Table 2 and Figure 3 (right). Table 2 also includes the $t_{0}$ from the comparison of mean values which proves that the use of priority-based control yields a significantly better performance compared to the random selection. 
Table 1. Shifted Sphere. Error values achieved for $N=10, N=30$ and $N=50$ design variables

\begin{tabular}{|c|c|c|c|c|}
\hline Evaluations & & $\mathrm{N}=10$ & $\mathrm{~N}=30$ & $\mathrm{~N}=50$ \\
\hline \multirow{7}{*}{1000} & 1st (best) & 19.6280 & 6969.9413 & 33551.0950 \\
\hline & 7 th & 60.7039 & 9957.6341 & 50590.2323 \\
\hline & 13th (median) & 94.5923 & 13490.6585 & 55570.7452 \\
\hline & 19 th & 187.8432 & 16027.9618 & 58077.7842 \\
\hline & 25th (worst) & 460.4852 & 23476.1358 & 71718.8583 \\
\hline & Mean & 139.7283 & 13745.0322 & 54829.2035 \\
\hline & Stdev & 113.6186 & 4580.4962 & 8866.3967 \\
\hline \multirow{7}{*}{10000} & 1st (best) & $7.2100 \mathrm{E}-08$ & 0.6900 & 49.9178 \\
\hline & 7 th & $8.0230 \mathrm{E}-07$ & 1.0033 & 93.9828 \\
\hline & 13th (median) & $1.0699 \mathrm{E}-06$ & 1.4833 & 129.8803 \\
\hline & 19 th & $2.2854 \mathrm{E}-06$ & 2.3223 & 201.9965 \\
\hline & 25 th (worst) & $2.4199 \mathrm{E}-06$ & 8.2761 & 1116.3599 \\
\hline & Mean & $3.0715 \mathrm{E}-06$ & 2.3484 & 205.1065 \\
\hline & Stdev & $5.2963 \mathrm{E}-06$ & 2.2694 & 221.8390 \\
\hline \multirow{7}{*}{100000} & 1st (best) & $1.0000 \mathrm{E}-15$ & $1.6416 \mathrm{E}-05$ & $1.8933 \mathrm{E}-03$ \\
\hline & 7 th & $3.0001 \mathrm{E}-10$ & $2.1874 \mathrm{E}-05$ & 3.3741E-03 \\
\hline & 13th (median) & $4.9999 \mathrm{E}-10$ & $3.2594 \mathrm{E}-05$ & $4.6081 \mathrm{E}-03$ \\
\hline & 19 th & $7.0002 \mathrm{E}-10$ & $4.1963 \mathrm{E}-05$ & $5.4220 \mathrm{E}-03$ \\
\hline & 25 th (worst) & $1.4999 \mathrm{E}-09$ & $2.5631 \mathrm{E}-04$ & $9.2563 \mathrm{E}-03$ \\
\hline & Mean & $5.0399 \mathrm{E}-10$ & $4.2348 \mathrm{E}-05$ & $4.5626 \mathrm{E}-03$ \\
\hline & Stdev & $3.4336 \mathrm{E}-10$ & 4.6663E-05 & $1.5576 \mathrm{E}-03$ \\
\hline \multirow{7}{*}{$\begin{array}{l}\text { End }(300000 \text { for } \\
\mathrm{N}=30 \text { or } 500000 \\
\text { for } \mathrm{N}=50)\end{array}$} & 1st (best) & - & $3.9460 \mathrm{E}-07$ & $2.0408 \mathrm{E}-04$ \\
\hline & 7 th & - & $1.3893 \mathrm{E}-06$ & 2.7999E-04 \\
\hline & 13th (median) & - & $1.9769 \mathrm{E}-06$ & $3.0998 \mathrm{E}-04$ \\
\hline & 19 th & - & $2.7324 \mathrm{E}-06$ & $3.6979 \mathrm{E}-04$ \\
\hline & 25 th (worst) & - & 1.6923E-04 & $5.8200 \mathrm{E}-04$ \\
\hline & Mean & - & $8.7464 \mathrm{E}-06$ & $3.3667 \mathrm{E}-04$ \\
\hline & Stdev & - & $3.3449 \mathrm{E}-05$ & $9.6978 \mathrm{E}-05$ \\
\hline
\end{tabular}
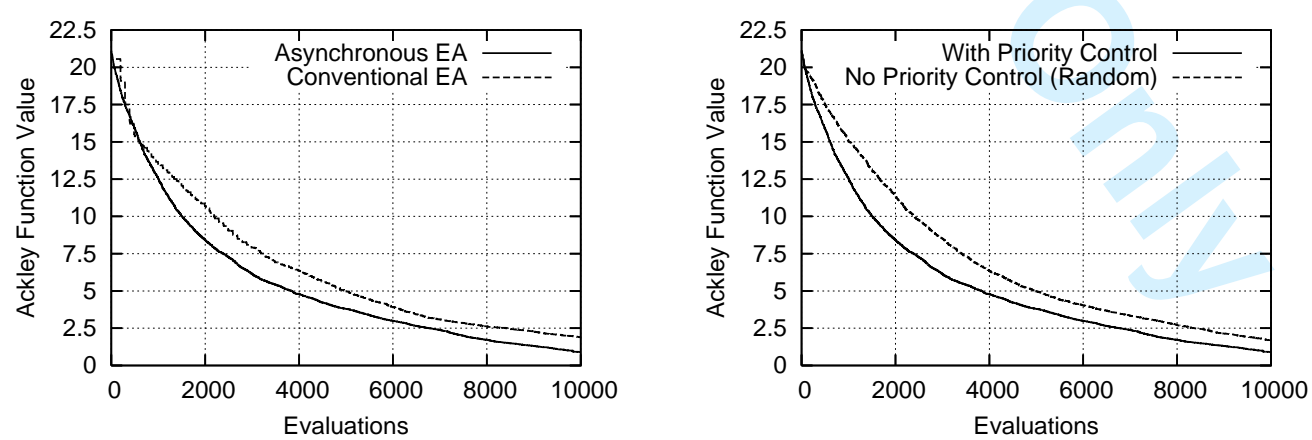

Figure 3. Minimization of the Ackley function. Average convergence history of the proposed AEA compared to a conventional EA (left); average convergence history of the AEA with and without priority control (right).

The next mathematical case considered is the two-objective minimization of ZDT3 function, (Zitzler et al. 2000), with $N=30$. This case produces a discon- 
Table 2. Minimization of the Ackley function. Comparison of AEAs with and without priority control.

\begin{tabular}{ccc}
\hline & $\begin{array}{c}\text { Controlled by } \\
\text { Priority }\end{array}$ & $\begin{array}{c}\text { Uncontrolled } \\
\text { (Random) }\end{array}$ \\
\hline$f_{\text {mean }}$ & 0.8963 & 1.6939 \\
$f_{\text {min }}$ & 0.1958 & 1.0416 \\
$f_{\text {max }}$ & 1.4394 & 2.3727 \\
$s$ & 0.3861 & 0.3447 \\
$t_{0}$ & 8.4386 & \\
\hline
\end{tabular}

tinuous Pareto front and was selected in order to study the interaction between demes during the optimization. The supporting mesh was $10 \times 10$ and with 25 poles it was challenging to compute a Pareto front split into five parts. 30 runs with 25000 evaluations each have been carried out using different RNG seeds and 18 CPUS (on the first and second cluster; heterogeneous platform). For all runs, the hypervolume indicator $\left(I_{H}\right)$ was computed with reference point $\left(f_{1}, f_{2}\right)=(5,3)$ and the mean value and standard deviation are shown in Table 3 . From the same table and the $t_{0}$ value obtained after a $\mathrm{t}$-test, we conclude that the proposed method outperforms the conventional EA. The "best" (according to the hypervolume indicator) Pareto fronts from the proposed AEA and the conventional EA are shown in Figure 4.

Table 3. Two-objective $Z D T 3$ case. Hypervolume computation and t-tests.

\begin{tabular}{ccc}
\hline & AEA & Conventional EA \\
\hline$I_{H_{\text {mean }}}$ & 18.0318 & 17.8584 \\
$s$ & 0.5106 & 0.4559 \\
$t_{0}$ & 13.8750 & \\
\hline
\end{tabular}
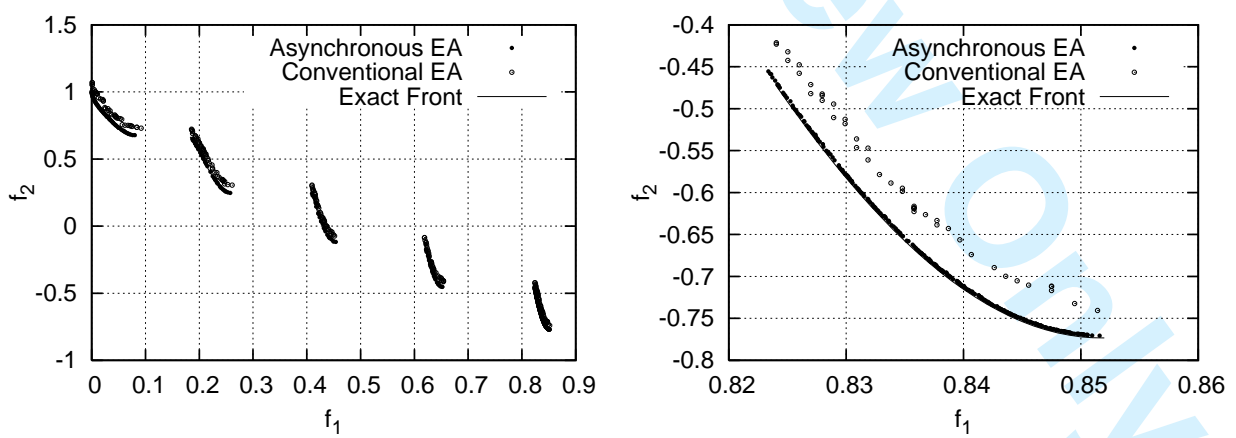

Figure 4. Two-objective ZDT3 case. Pareto front approximations computed by the proposed AEA and the conventional EA after 25000 evaluations compared to the exact Pareto front. A close- up view of the right-most sub-front is shown on the right.

In the same case, the Pareto front evolution was also studied. For one of the RNG seeds, the fronts of non-dominated solutions as well as the position of poles, after 3000, 7000, 10000, 15000, 20000 and 25000 evaluations, are plotted in Figure 5 . Note that, due to the plotting bounds, some of the 25 poles cannot be shown. On the same instants during the evolution, the numbers of currently non-dominated solutions produced by each of the 25 demes are plotted in Figure 6 . We may 
conclude that the interaction between demes is adequate (this is, in fact, a proof that these are "strongly interacting demes" as mentioned in the introduction) since on various instants of the evolution the non-dominated solutions are produced by different demes in a continuously varying percentage. For instance, pole 1 generated non-dominated solutions on four sub-fronts though it lies on one of them.
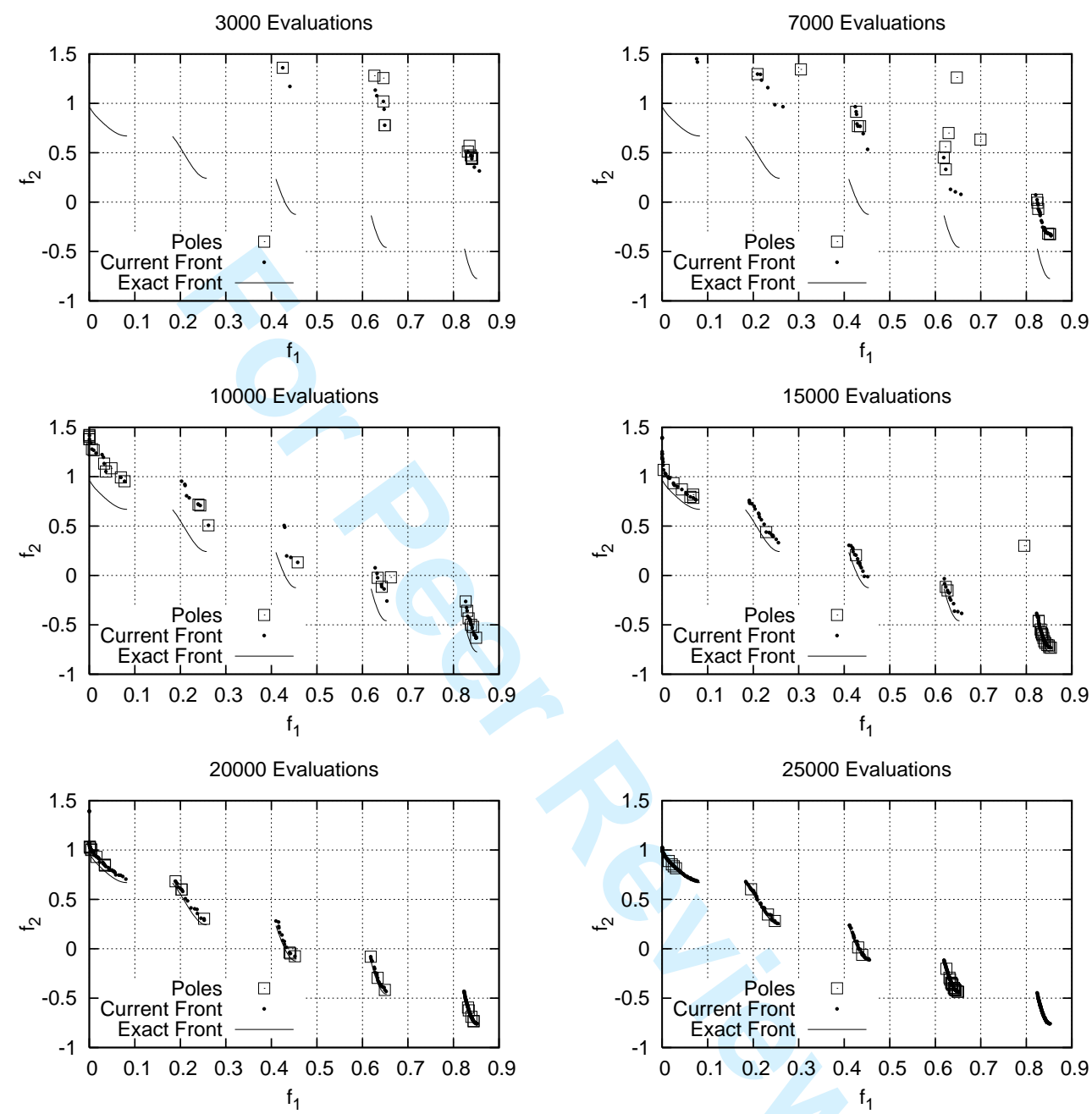

Figure 5. Two-objective ZDT3 case. Evolution of the front of non-dominated solutions during the optimization. Poles position is also shown.

The last numerical application is the constrained design of a cylindrical pressure vessel capped at both ends by hemispherical heads. This problem was first presented and solved by Sandgren (Sandgren 1990) using a branch and bound technique. A variety of solution methods have been tested on the same case, including ALM (Kannan and Kramer 1994), GeneAS (Deb 1997), GA with tournament selection (Coello and Montes 2002), $\epsilon$ constrained Differential Evolution $(\epsilon \mathrm{DE})$ (Takahama et al. 2006) and co-evolutionary particle swarm (CPSO) (He and Wang 2007). The objective is to minimize $f(\vec{x})=0.6224 x_{1} x_{3} x_{4}+1.7781 x_{2} x_{3}^{2}+$ $3.1661 x_{1}^{2} x_{4}+19.84 x_{1}^{2} x_{3}$ which includes the cost of the material, forming and welding. The problem has $N=4$ design variables, namely the thicknesses of shell $\left(x_{1}\right)$ and head $\left(x_{2}\right)$, the inner radius $\left(x_{3}\right)$ and the length of the cylindrical section of the vessel $\left(x_{4}\right) . x_{1}$ and $x_{2}$ take on values that are integer multiples of $0.0625 \mathrm{in}$, corresponding to the available thicknesses of rolled steel plates, whereas $x_{3}$ and $x_{4}$ are continuous variables with $1<x_{1,2}<99$ and $10<x_{3,4}<200$. Four geometrical constrains were imposed. 30 optimizations were carried out with 10000 

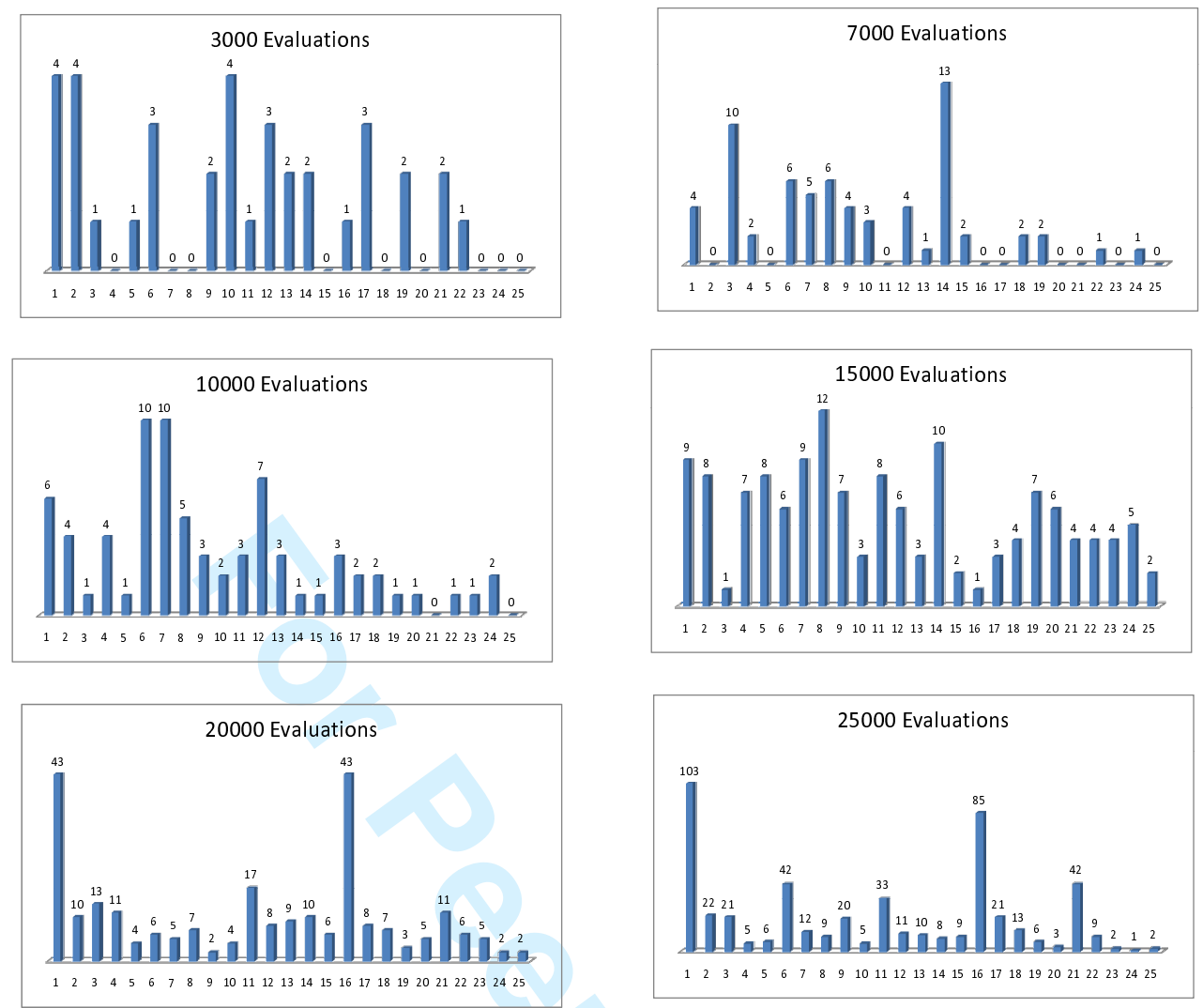

Figure 6. Two-objective ZDT3 case. Number of members of the front of non-dominated solutions produced by each deme after 3000, 7000, 10000, 15000, 20000 and 25000 evaluations.
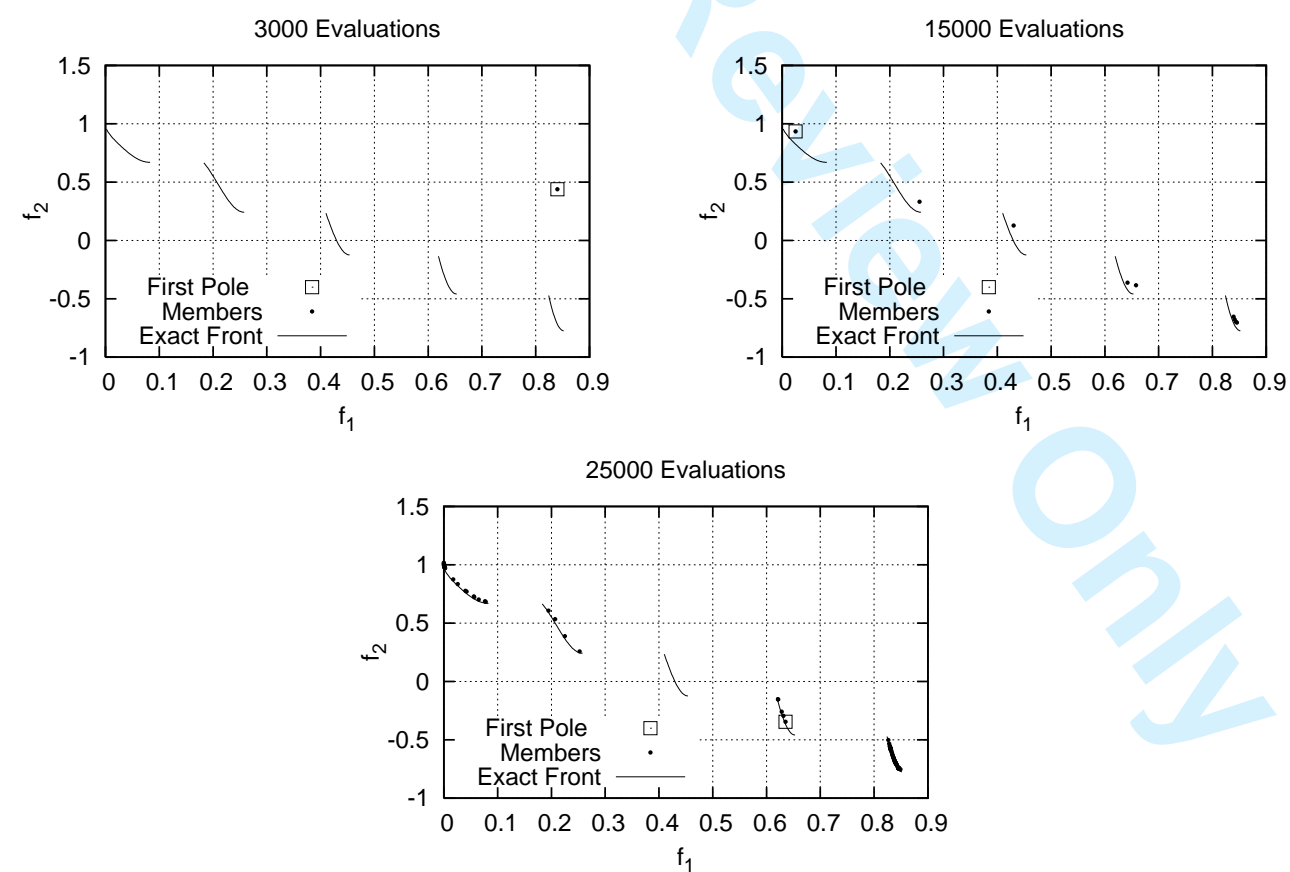

Figure 7. Two-objective ZDT3 case. Non-dominated members generated from deme 1 and the corresponding pole after 3000, 15000 and 25000 evaluations.

evaluations each using various RNG numbers and supporting mesh sizes, on 20 CPUS (of the first and second cluster). The mean optimal solution corresponds to $f_{\text {mean }}=6116.008$ (worst $f_{\max }=6410.153$, best $\left.f_{\min }=6059.714\right)$. The average 
convergence of the 30 optimization runs is plotted in Figure 8 (for the sake of clarity this plot is limited to 5000 evaluations). Table 4 compares some of the "optimal" solutions found in the literature; in this table, the constraints' values are given considering that all constraints were cast in the form $c_{i}(\vec{x}) \leq 0$ (some of the presented "optimal" solutions were, thus, infeasible). From this table, it can be seen that the best solution found by the proposed AEA is better than the best solutions found by other techniques and only slightly inferior than the one of $\epsilon \mathrm{DE}$ (Takahama et al. 2006). We may also comment on the number of evaluations needed to achieve this solution. AEA and $\epsilon \mathrm{DE}$ were run for 10000 evaluations each while $\mathrm{CPSO}$ (He and Wang 2007) for 200000 and GA (Coello and Montes 2002) for 80000. Finally, in a direct comparison of $\mathrm{AEA}$ with $\epsilon \mathrm{DE}$, the proposed algorithm produces constantly good solutions after the first 2000 evaluations instead of the 5000 required by $\epsilon \mathrm{DE}$ (Takahama et al. 2006) for the same quality of solution.

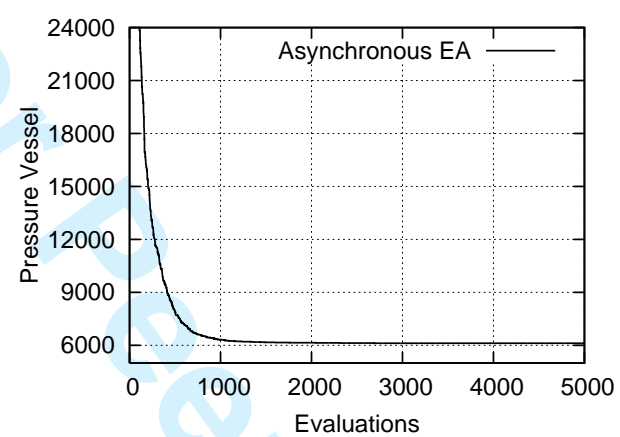

Figure 8. Design of an optimal pressure vessel: Average convergence history of 30 optimization runs. This plot stops at 5000 evaluations, though 10000 evaluations have been carried out.

Table 4. Design of a pressure vessel: Comparison of optimal solutions.

\begin{tabular}{cccccccc}
\hline $\begin{array}{c}\text { Method } \\
\text { Year }\end{array}$ & AEA & CPSO & $\epsilon$ DE & GA & GeneAS & ALM & $B \& B$ \\
& 2008 & 2007 & 2006 & 2002 & 1997 & 1994 & 1988 \\
\hline$x_{1}$ & 0.8125 & 0.8125 & $N / A$ & 0.8125 & 0.9375 & 1.1250 & 1.1250 \\
$x_{2}$ & 0.4375 & 0.4375 & $N / A$ & 0.4375 & 0.5000 & 0.6250 & 0.6250 \\
$x_{3}$ & 42.0984 & 42.091266 & $N / A$ & 42.097398 & 48.3290 & 58.291 & 47.700 \\
$x_{4}$ & 176.6366 & 176.7465 & $N / A$ & 176.65405 & 112.6790 & 43.6900 & 117.701 \\
$c_{1}(\vec{x})$ & $-4.310^{-8}$ & -0.000139 & $N / A$ & -0.00002 & -0.004750 & 0.000016 & -0.20439 \\
$c_{2}(\vec{x})$ & -0.03588 & -0.035949 & $N / A$ & -0.035891 & -0.038941 & -0.068904 & -0.169942 \\
$c_{3}(\vec{x})$ & -0.000052 & -116.3827 & $N / A$ & -27.886075 & -3652.876 & -21.220104 & 54.226012 \\
$c_{4}(\vec{x})$ & -63.3634 & -63.2535 & $N / A$ & -63.345953 & -127.3210 & -196.3100 & -122.29900 \\
\hline \multirow{2}{*}{$f(\vec{x})$} & $\mathbf{6 0 5 9 . 7 1 4 6}$ & 6061.0777 & 6059.7143 & 6059.9463 & 6410.3811 & 7198.0428 & 8129.1036 \\
\hline
\end{tabular}

\subsection{Design of a 2D Compressor Cascade}

The first aerodynamic optimization problem is concerned with the constrained design of a $2 \mathrm{D}$ compressor cascade for minimum total pressure loss coefficient $\omega=\left(p_{t_{1}}-p_{t_{2}}\right) / 0.5 \rho_{1} V_{1}^{2}$, where $p_{t}$ is the total pressure, $\rho$ the density and $V$ the flow velocity. Indices 1 and 2 indicate the inlet to and outlet from the cascade, 
respectively. The cascade was designed to operate at isentropic exit Mach number $M_{2, i s}=0.45$, inlet flow angle $a_{1}=47^{\circ}$ and Reynolds number based on chord $R e_{c}=840000$. It has fixed stagger angle $30^{\circ}$, solidity $\sigma=0.68$ and axial-velocitydensity ratio equal to 0.89 . The airfoil shape was parameterized using Bézier curves with 18 control points ( 9 on each side). All but the leading and trailing edges control points were allowed to vary in both chordwise and normal-to-the-chord direction, summing up to 28 design variables. The evaluation tool was an in-house NavierStokes flow solver (vertex-centered, finite--volume scheme with a second-order upwind scheme for the convection terms) for compressible flows on unstructured grids (Lambropoulos et al. 2004), coupled with the Spalart-Allmaras (Spalart and Allmaras 1994) turbulence model. For CFD computations, unstructured grids with triangular elements, are used. These are generated using the advancing front technique and superimposed to structured-like layers of triangles (stretched rectangular mesh whose elements split into triangles) surrounding the cascade. To prevent the formation of unacceptably thin airfoils, geometrical constraints on the airfoil thickness $T$ at three chordwise positions

$$
T(0.30 c) \geq 0.08 c, T(0.60 c) \geq 0.07 c, T(0.90 c) \geq 0.01 c
$$

where $c$ is the chord length, were imposed. Should any of the geometrical constraints be violated, cascade does not further undergo flow analysis. With respect to Eq. 3, the first constraint $(j=1)$ gives $c_{1}=-T(0.30 c), c_{1}^{\text {thres }}=-0.08 c$ and $c_{1}^{\text {dead }}=0.80 c_{1}^{\text {thres }}$. The same ratio $c_{j}^{\text {dead }} / c_{1}^{\text {thres }}=0.80$ was used for all constraints. Also, $\alpha=3$. Due to this filter, the CPU cost per evaluation varies between almost zero (for infeasible-thin airfoils; no Navier-Stokes solution) and the cost of getting a converged Navier-Stokes solution. Note, also, that even the cost of a Navier-Stokes based evaluation may vary noticeably. Since unstructured grids are generated, the grid size per individual (and thus, the CPU cost per iteration) may differ. Also, the number of iterations to obtain a converged solution may also vary a lot depending on the flow phenomena to be modeled. Apart from geometrical constraints, a minimum flow turning angle constraint was also imposed. In contrast to the geometrical constraints, this should be applied only after solving the flow equations. The single-objective optimization was repeated four times with different supporting mesh dimensions, namely $6 \times 6,8 \times 8,10 \times 10$ and $12 \times 12$ allowing 2000 calls of the evaluation software, on $20 \mathrm{CPUs}$ (third cluster). The best result found was $\omega=0.0131$ on the $8 \times 8$ supporting mesh. The convergence history from all runs performed and the optimal airfoil contour found are plotted in Figure 9 . The optimal solutions are very close to each other (vary from $\omega=0.0131$ on the $8 \times 8$ mesh to $\omega=0.01333$ on the $10 \times 10$ mesh). From the convergence history, it can be seen that the $8 \times 8$ and $10 \times 10$ runs progress faster during the first 300 evaluations.
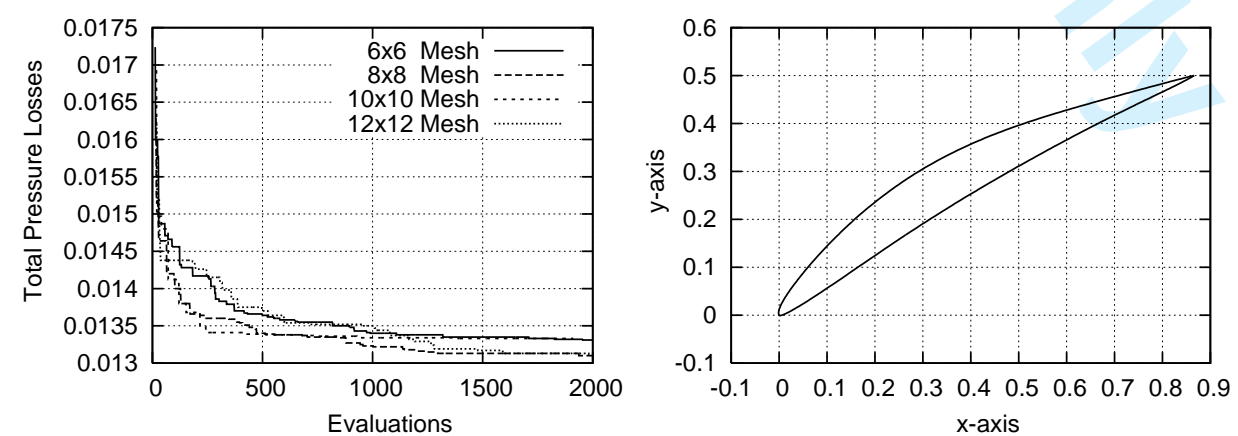

Figure 9. Design of a 2D compressor cascade. Optimization history for all tested supporting mesh sizes (left); optimal airfoil contour (right). 
This case was also used to compare the proposed AEA to a conventional EA in terms of the wall clock time needed for an optimization. For such a comparison, the optimization runs were also repeated several times with different RNG numbers. Since an optimization using a Navier-Stokes flow solver is time-consuming, a viscous-inviscid flow interaction method with an integral boundary layer solver (Drela and Giles 1987) was used instead. All runs were performed on the third cluster using 8 CPUs. The populations for the conventional EA were multiples of $8((\mu, \lambda)=(64,128)$, where $\mu, \lambda$ are the numbers of parents and offspring, respectively) so as to theoretically guarantee the full utilization of all processors. In practice, however, this is not the case because of the imposed geometrical constraints which may lead to idle processor times during each generation. For the proposed AEA, three supporting mesh sizes $(8 \times 8,10 \times 10$ and $12 \times 12)$ were used. The runs were performed using 3 different RNG values and 5000 evaluations. Each run of the AEA, on each supporting mesh, was repeated three times and, then, averaged. The results are presented in Table 5. The different number of performed evaluations in each case is due to the geometrical constraints (only flow evaluations are counted; rejecting a candidate solution during checking for the geometrical constraints is not considered to be an evaluation). In all cases, the AEA is constantly more efficient compared to the conventional EA. Figure 10 compares the convergence history of optimizations with different RNG numbers. The convergence shown for each supporting mesh size of the AEA is the average one of the three optimizations performed for the given mesh and RNG number.

Table 5. Design of a 2D compressor cascade. Comparison of the AEA (three configurations) with a conventional EA. Wall clock time is measured in minutes.

\begin{tabular}{ccccc}
\hline & & RNG1 & RNG2 & RNG3 \\
& & Evals / Time & Evals / Time & Evals / Time \\
\hline Conventional EA & $(64,128)$ & $4864 / 31$ & $4845 / 29.5$ & $4965 / 30$ \\
AEA & $8 \times 8$ & $4758 / 28$ & $4728 / 27.5$ & $4802 / 28$ \\
& $10 \times 10$ & $4735 / 27.5$ & $4751 / 28$ & $4758 / 28.5$ \\
& $12 \times 12$ & $4705 / 28$ & $4750 / 27.5$ & $4732 / 28$ \\
\hline
\end{tabular}

A last study was related to the effect of number of the available CPUs. The $10 \times 10$ supporting mesh $\left(N_{\text {agents }}=75, N_{\text {poles }}=25\right)$ was used (for RNG1). We tested $N_{C P U}$ 4, 6, 8, 15 and 24 CPUs and the results are presented in Figure 11. By increasing the number of CPUs, the algorithm is capable of finding better solutions within the same CPU time. Table 6 gives the wall clock time of optimization runs with up to 24 processors. The stopping criterion was the same number of evaluations for all of them. Parallel speed-up values are also given by matching the assumption that the CPU cost on a single processor was $53.5 \times 4=214 \mathrm{~min}$ and also, that (despite small variations) the number of evaluations was exactly the same. We recall that this is an approximate parallel speed-up since the CPU cost per evaluation was not constant (although the computations were made on an homogeneous platform). However, a speed-up close to 23 for 24 processors is extremely satisfactory.

\subsection{Design of an Isolated Airfoil}

The second aerodynamic case is a two-objective, constrained, optimization problem which is concerned with the design of an isolated airfoil for minimum drag 


\section{Engineering Optimization}

Engineering Optimization

1

7

8

9

10

11

12

13

14

15

16

17

18

19

20

21

22

23

24

25

26

27

28

29

30

31

32

33

34

35

36

37

38

39

40

41

42

43

44

45

46

47

48

49

50

51

52

53

54

55
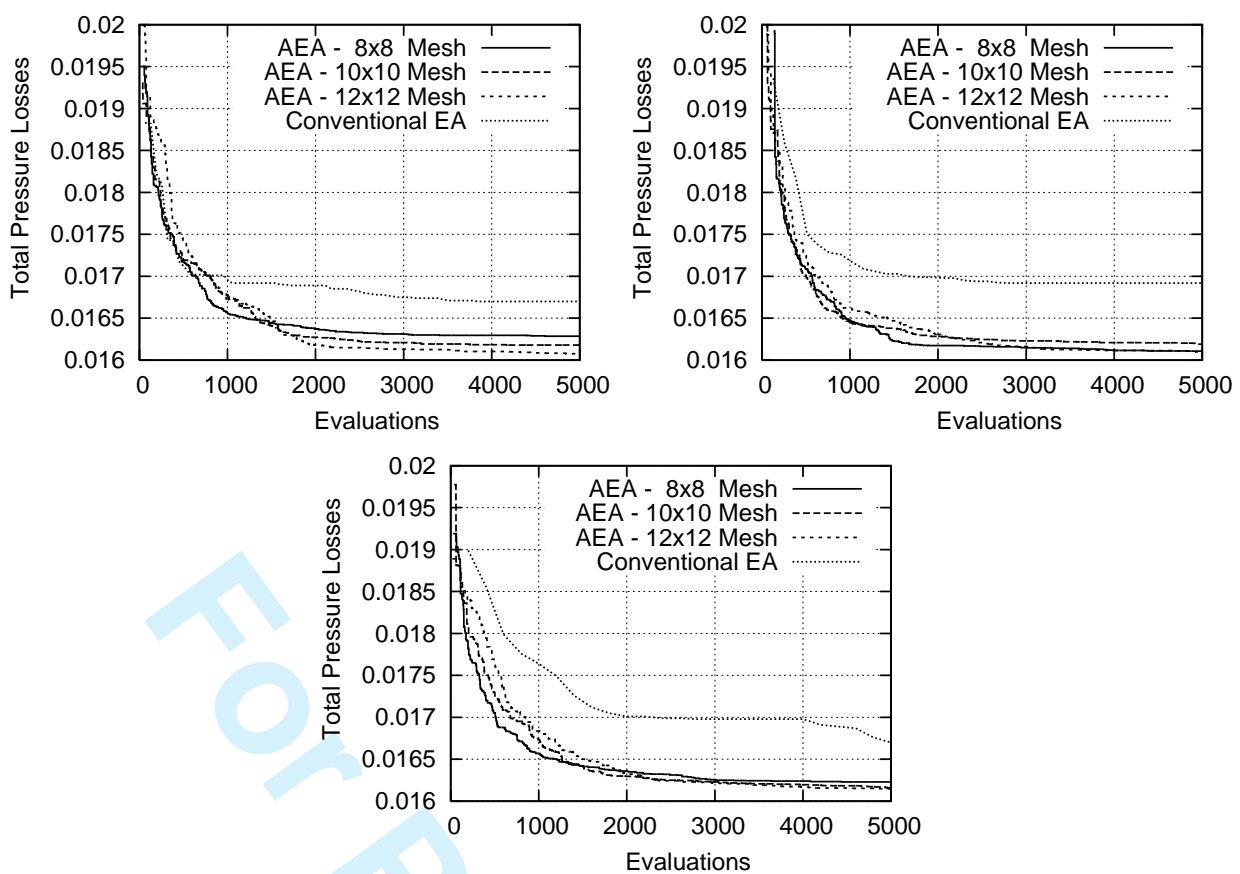

Figure 10. Design of a 2D compressor cascade. Comparison of the convergence history of AEA (three different mesh sizes) and conventional EA for RNG1 (up-left), RNG2 (up-right) and RNG3 (down).
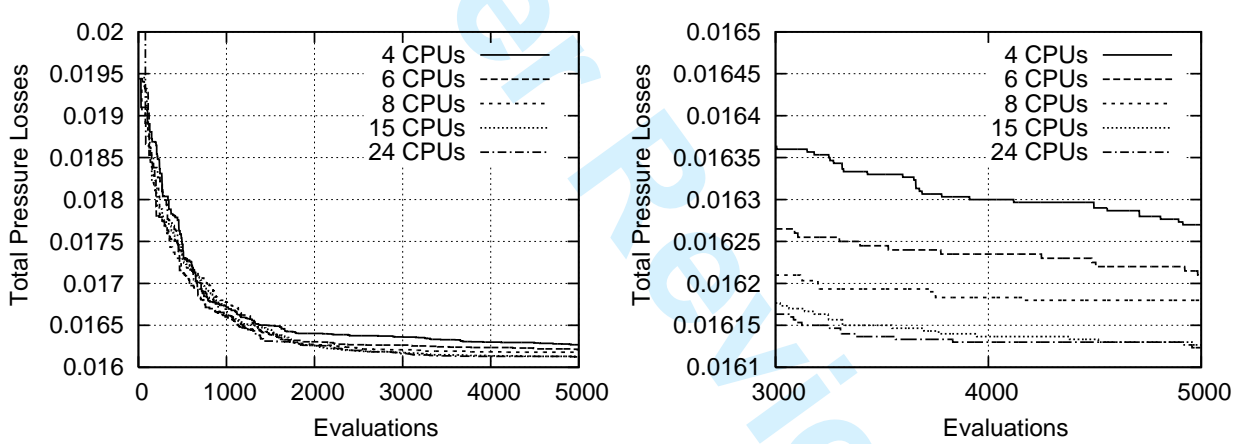

Figure 11. Design of a 2D compressor cascade. Effect of the number of the available CPUs on the optimal solution, during the whole evolution (left) or by focusing on the last 2000 evaluations (right).

Table 6. Design of a 2D compressor cascade. Wall clock time and estimated parallel speed-up according to the number of CPUs.

CPUs Wall Clock Time Evaluations Parallel Speed-up

\begin{tabular}{cccc}
\hline 4 & $53.5 \mathrm{~min}$ & 4752 & 4 \\
6 & $38 \mathrm{~min}$ & 4750 & 5.6 \\
8 & $28 \mathrm{~min}$ & 4758 & 7.6 \\
15 & $15 \mathrm{~min}$ & 4758 & 14.3 \\
24 & $9.5 \mathrm{~min}$ & 4741 & 22.5 \\
\hline
\end{tabular}

coefficient $\left(C_{D}\right)$ and maximum lift coefficient $\left(C_{L}\right)$. The flow conditions were: freestream Mach number $M_{\infty}=0.15$, flow angle $a_{\infty}=6^{\circ}$ and $R e_{c}=3 \times 10^{6}$. The airfoil was parameterized using two Bézier curves, separately for the pressure and suction sides, with 7 control points each, concluding to 20 design variables. Constraints on 
the airfoil thickness at given chordwise positions were imposed, namely

$$
T(0.15 c) \geq 0.06 c, T(0.35 c) \geq 0.08 c, T(0.60 c) \geq 0.07 c, T(0.85 c) \geq 0.02 c
$$

These constraints were handled as in the cascade (see Eq. 12 and the subsequent comments). The evaluation software was the same Navier-Stokes solver used in the previous study; unstructured grids were used. This case was also studied for supporting meshes of different sizes and a stopping criterion of 2000 evaluations. 24 CPUs, of the second and third cluster, were used. The Pareto fronts obtained from all runs are presented in Figure 12, left. From this figure, it's not obvious which Pareto front approximation is better. So, the overall non-dominated solutions among all front approximations are found and plotted in the right side of Figure 12. This Pareto comprises 150 members, originating as follows: 74 from the $6 \times 6$ mesh, 56 from the $8 \times 8$ mesh, 1 from the $10 \times 10$ mesh and 19 from the $12 \times 12$ mesh. According to this, the $6 \times 6$ mesh seems to perform better. Three solutions (airfoil shapes) from this computation are shown in Figure 13.
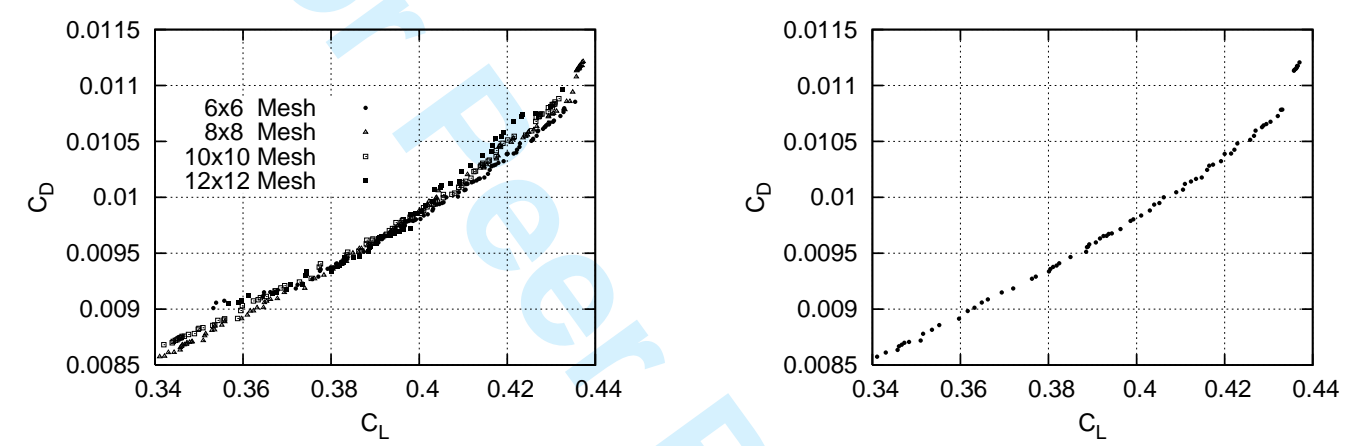

Figure 12. Design of an isolated airfoil. "Pareto" fronts resulting from runs with different supporting mesh sizes (left); overall non-dominated solutions from the comparison of all "Pareto" fronts (right).

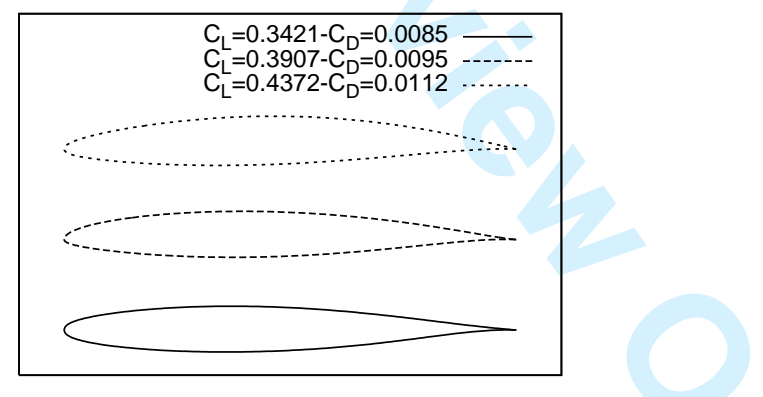

Figure 13. Design of an isolated airfoil. Selected airfoil shapes from the Pareto front of non-dominated solutions.

\section{Conclusions}

A parallel asynchronous evolutionary algorithm controlled by strongly interacting demes was presented. Single- and multi-objective mathematical and aerodynamicturbomachinery optimization problems, were used to assess the proposed method. Comparisons with conventional EAs and other results in literature were performed.

The proposed algorithm fully exploits the available CPUs and is faster compared to a conventional EA in terms of wall clock time. Furthermore, in all cases studied, AEA is constantly better than the conventional EA. 
The effects of priority control, supporting mesh size and number of CPUs used have also been studied. The priority control proved to be an important issue and its absence leads to worst results. For the examined problems, the use of supporting mesh sizes less or equal to approximately $10 \times 10$ are recommended. The use of as many processors as possible (less processors than the size of supporting mesh, of course) is also recommended, since the asynchronous algorithm maximizes the exploitation of all available processors with no idle periods of time (no synchronization barrier). Increasing the number of CPUs, the wall clock time reduces (for the same quality of achieved solution) with an excellent parallel speed-up.

\section{References}

Ackley, D.H., 1987. A connectionist machine for genetic hillclimbing. Boston, Kluwer Academic Publishers. Alba, E., Troya, J.M, 2001. Analyzing synchronous and asynchronous parallel distributed genetic algorithms. Future Generation Computer Systems, 4, 451-465.

Alba, E., Tomassini, M., 2002. Parallelism and evolutionary algorithms. IEEE Transactions on Evolutionary Computation, 6, 443-462.

Alba, E., Nebro, A.J., Troya, J.M, 2002. Heterogeneous computing and parallel genetic algorithms. Parallel and Distributed Computing, 62, 1362-1385.

Bäck, T., 1996. Evolutionary algorithms in theory and practice. Oxford University Press.

Cantú-Paz, E., 1998. A survey of parallel genetic algorithms. Calculateurs Paralleles, Reseaux et Systemes Repartis, 10, 141-171.

Coello, C.A.C., Montes, E.M., 2002. Constraint-handling in genetic algorithms through the use of dominance-based tournament selection. Advanced Engineering Informatics, 16, 193-203.

Deb, K., 1997. GeneAS, a robust optimal design technique for mechanical component design. Evolutionary algorithms in engineering applications. Springer, Berlin.

Drela, M., Giles, M.B., 1987. Viscous-Inviscid Analysis of transonic and low Reynolds number airfoils. AIAA Journal, 25, 1347-1355.

He, Q., Wang, L., 2007. An effective co-evolutionary particle swarm optimization for constrained engineering design problems. Engineering Applications of Artificial Intelligence, 20, 89-99.

Giannakoglou, K.C., 2002. Design of optimal aerodynamic shapes using stochastic optimization methods and computational intelligence. Progress in Aerospace Sciences, 38, 43-76.

Giannakoglou, K.C., Kampolis, I.C., Liakopoulos, P.I.K., Karakasis, M.K., Papadimitriou, D.I., Asouti, V.G., Zervogiannis, T., 2007. Aerodynamic shape optimization methods on multiprocessor platforms. International Conference on Parallel Computational Fluid Dynamics (Parallel CFD 2007), Antalya, Turkey, May, 2007.

Kannan, B.K., Kramer, S.N., 1994. An augmented Lagrange multiplier based method for mixed integer discrete continuous optimization and its applications to mechanical design. ASME Journal of Mechanical Design, 116, 318-320.

Kampolis, I.C., Zymaris, A.S., Asouti, V.G., Giannakoglou, K.C., 2007. Multilevel optimization strategies based on metamodel-assisted evolutionary algorithms, for computationally expensive problems. IEEE Congress on Evolutionary Computation, CEC 2007, 4116-4123, Singapore, 2007.

Kampolis, I.C., Giannakoglou, K.C., 2008. A multilevel approach to single-- and multiobjective aerodynamic optimization. Computer Methods in Applied Mechanics and Engineering, to appear.

Karakasis, M.K., Giannakoglou, K.C., 2006. On the use of metamodel-assisted multi-objective evolutionary algorithms. Engineering Optimization, 38, 941-957.

Lambropoulos, N.K., Koubogiannis, D.G., Giannakoglou, K.C., 2004. Acceleration of a Navier-Stokes equation solver for unstructured grids using agglomeration multigrid and parallel processing. Computer Methods in Applied Mechanics and Engineering, 193, 781-803.

Liakopoulos, P.I.K., Kampolis, I.C., Giannakoglou, K.C., 2008. Grid enabled, hierarchical distributed metamodel assisted evolutionary algorithms for aerodynamic shape optimization. Future Generation Computer Systems, 24, 701-708.

Lim, D., Ong, Y.S., Jin, Y., Sendhoffb, B., Lee, B.S., 2007. Efficient hierarchical parallel genetic algorithms using grid computing. Future Generation Computer Systems, 23, 658-670.

Manderick, B., Spiessens, P., 1989. Fine-grained parallel genetic algorithm. Proceedings of the third International Conference on Genetic Algorithms, 428-433.

Melab, N., Cahon, S., Talbi, E.G., 2006. Grid computing for parallel bioinspired algorithms. Journal of Parallel and Distributed Computing, 8, 1052-1061.

Michalewicz, Z., 1994. Genetic algorithms + data structures = evolutionary programs. Springer-Verlag, Berlin Heidelberg.

Muhammad, A., Bargiela, A., King, G., 1997. Fine-grained Parallel Genetic Algorithm: A Stochastic Optimisation Method. Proceedings of The First World Congress on Systems Simulation, 199-203.

Nowostawski, M., Poli, R., 1999. Parallel genetic algorithm taxonomy. Proceedings of the Third International Conference on Knowledge-based Intelligent Information Engineering Systems KES'99, 88-92.

Sandgren, E., 1990. Nonlinear integer and discrete programming in mechanical design optimization. $A S M E$ Journal of Mechanical Design, 112, 223-229.

Spalart, P., Allmaras, S., 1994. A one-equation turbulence model for aerodynamic flows. La Recherche Aerospatiale, 1, 5-21. 
Storn, R., Price, K., 1997. Differential Evolution - A simple and efficient heuristic for global optimization over continuous spaces. Journal of Global Optimization, 11, 341-359.

Suganthan, P.N., Hansen, N., Liang, J.J., Deb, K., Chen, Y.P., Auger, A., Tiwari, S., 2005. Problem definitions and evaluation criteria for the CEC 2005 special session on real-parameter optimization. Technical Report, Nanyang Technological University, Singapore And KanGAL Report Number 2005005 (Kanpur Genetic Algorithms Laboratory, IIT Kanpur)

Takahama, T., Sakai, S., Iwane, N., 2006. Solving nonlinear constrained optimization problems by the $\epsilon$ constrained differential evolution. IEEE International Conference on Systems, Man, and Cybernetics, $2322-2327$.

Zitzler, E., Deb, K., Thiele, L., 2000. Comparison of multiobjective evolutionary algorithms: Empirical results. Evolutionary Computation, 8, 173-195.

Zitzler, E., Laumanns, M., Thiele, L., 2002. SPEA2: Improving the strength pareto evolutionary algorithm for multiobjective optimization. Evolutionary Methods for Design, Optimisation and Control with Appllication to Industrial Problems (EUROGEN 2001), 95-100. International Center for Numerical Methods in Engineering (CIMNE). 


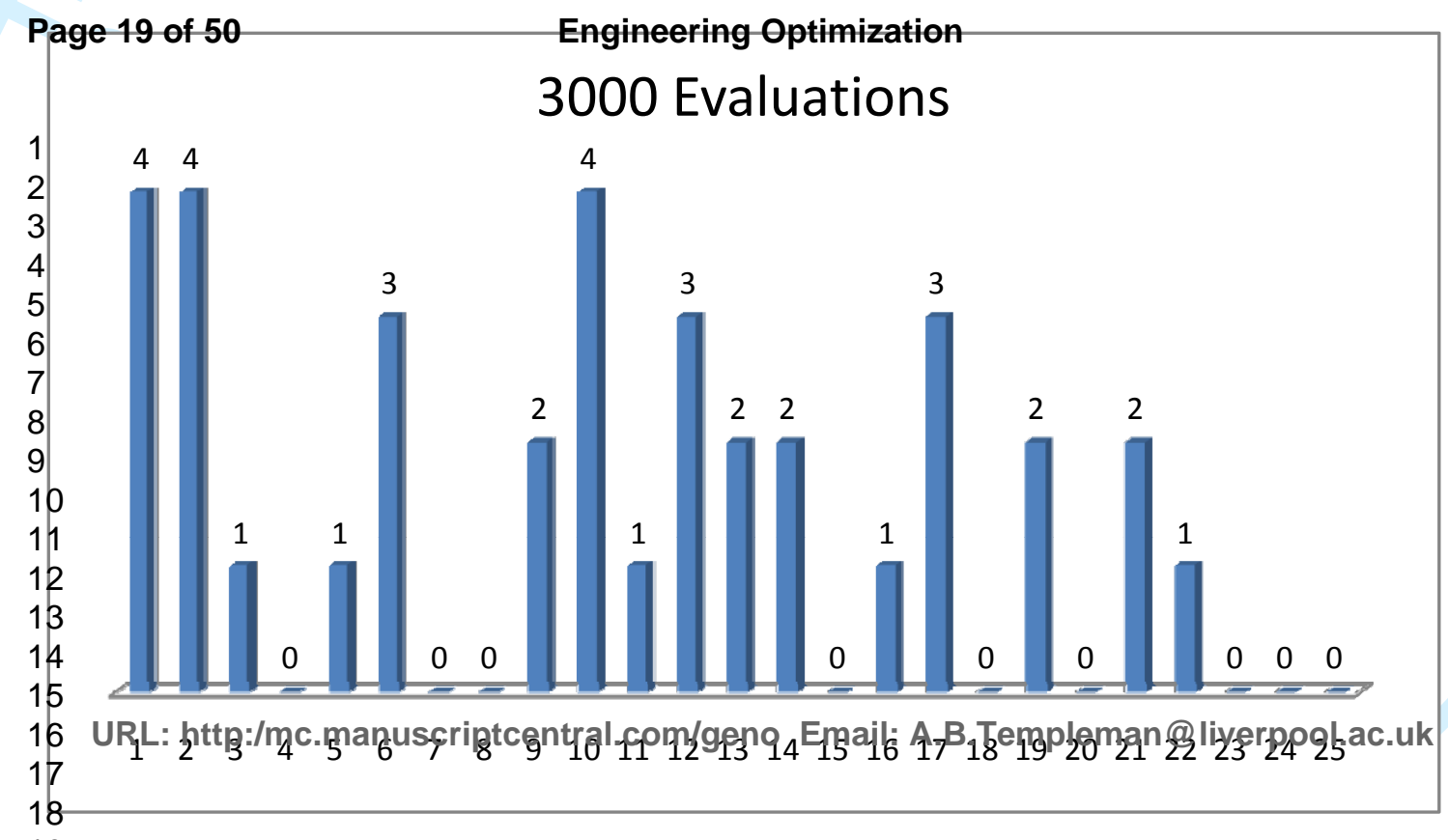




\section{Engineering Optimization 7000 Evaluations}

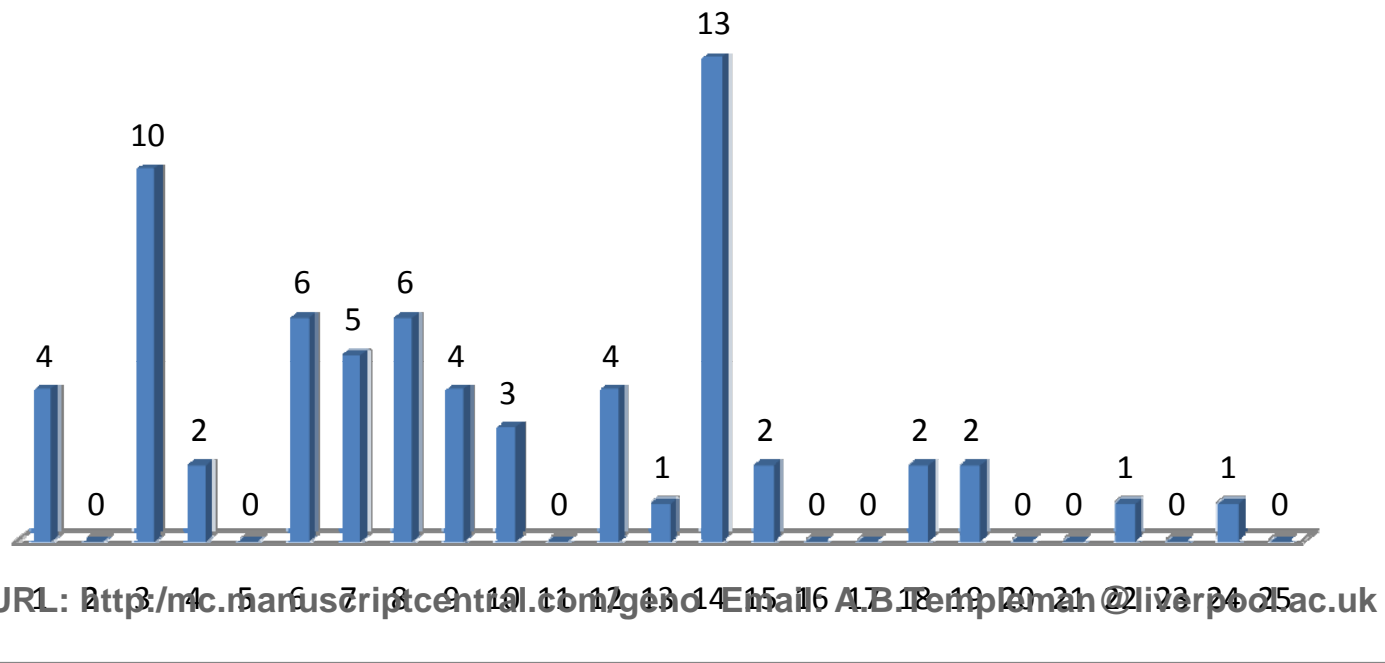

13

3

4

5

6 


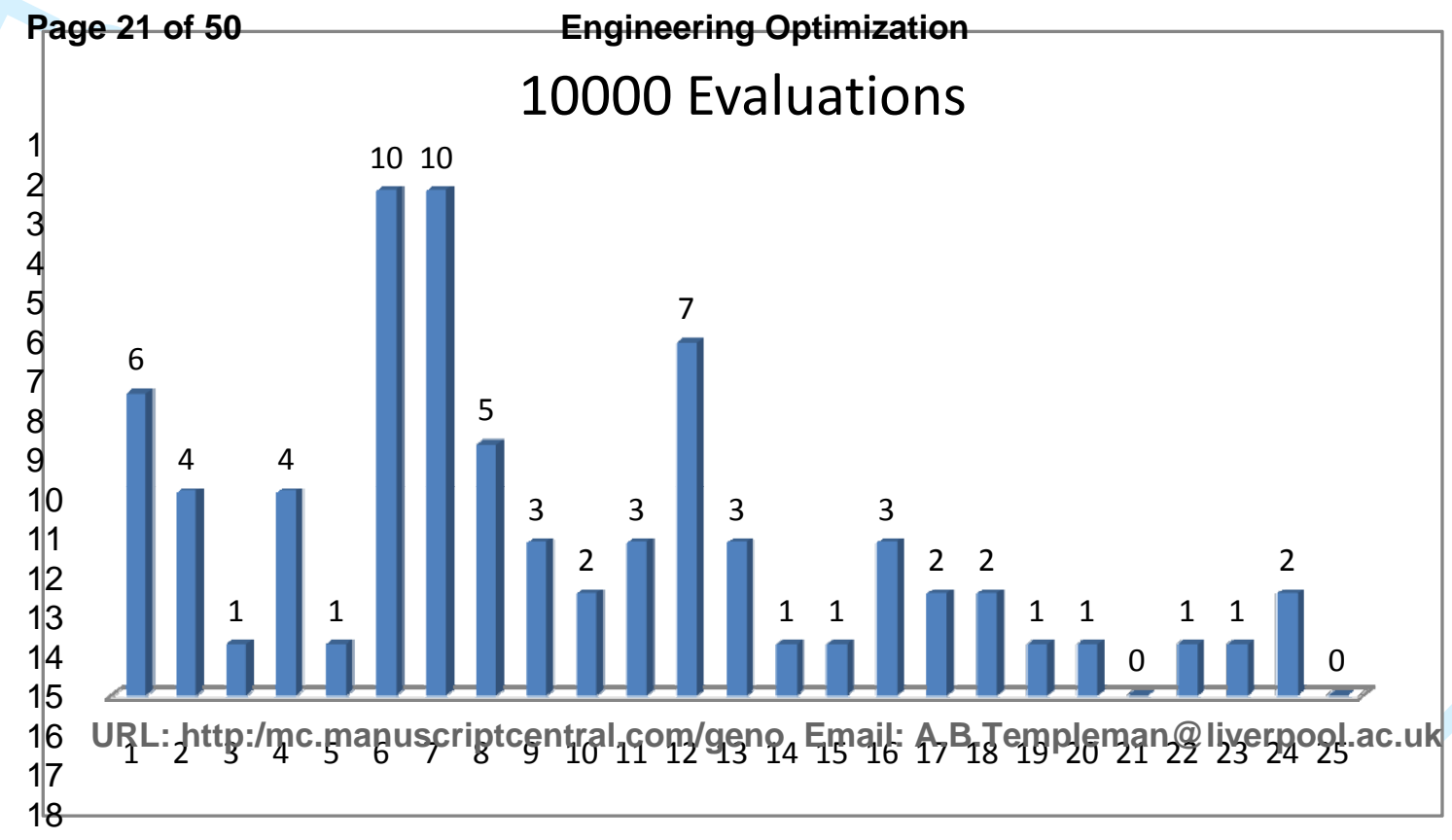




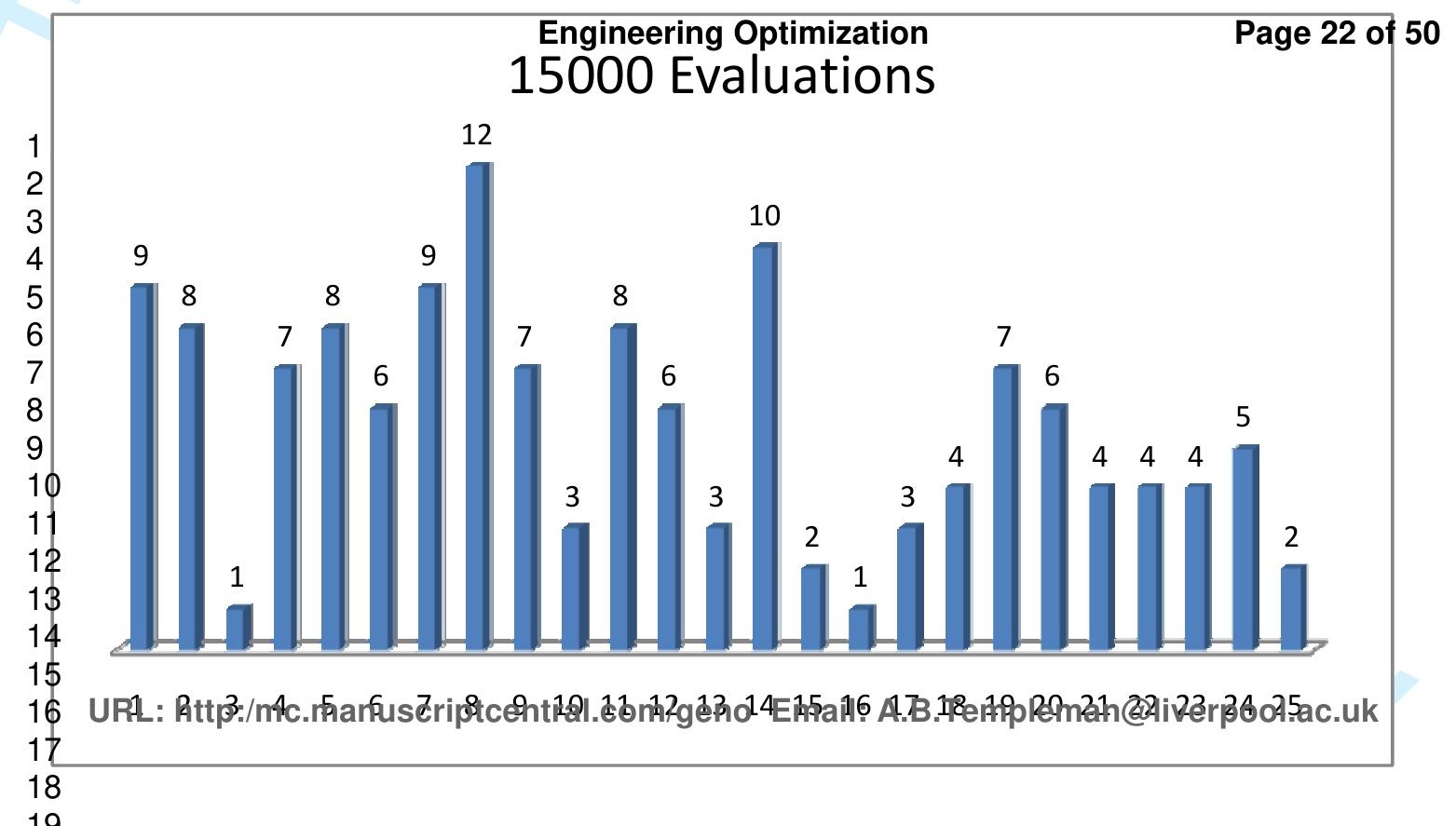




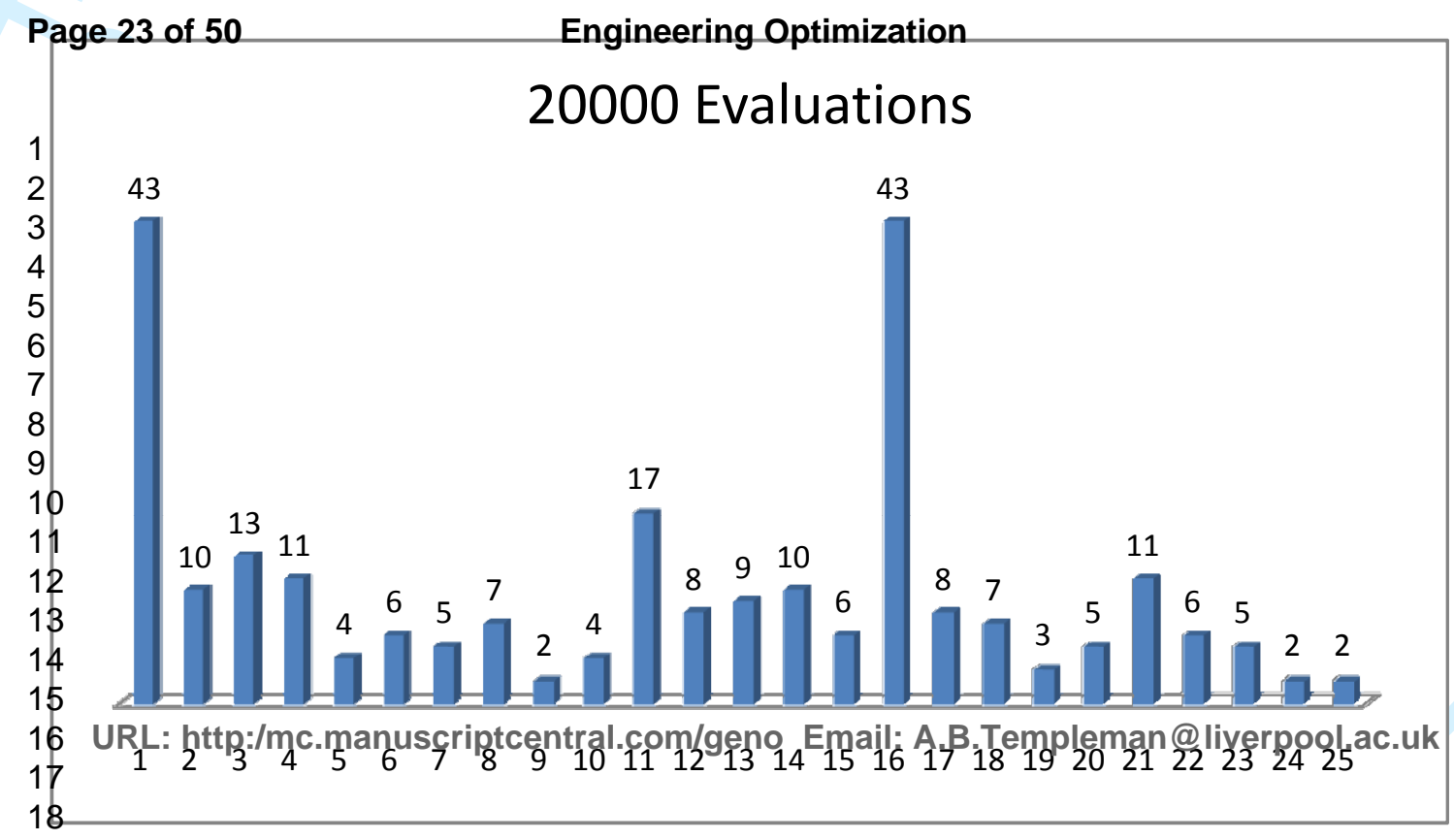




\section{Engineering Optimization 25000 Evaluations}

103

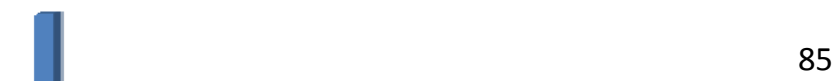

6

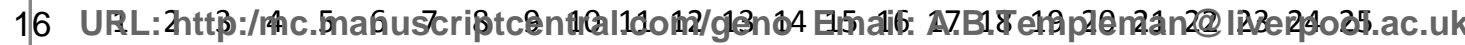
17 


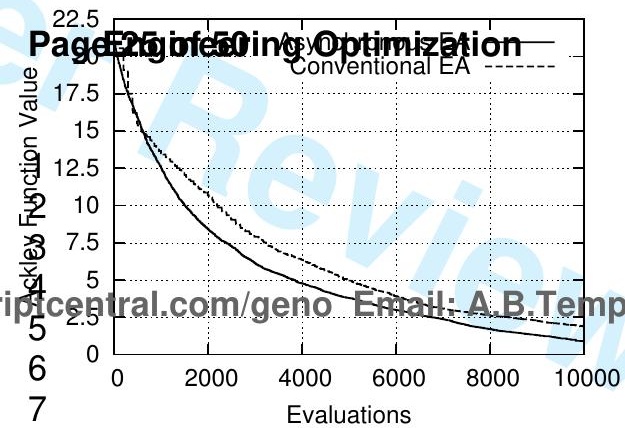




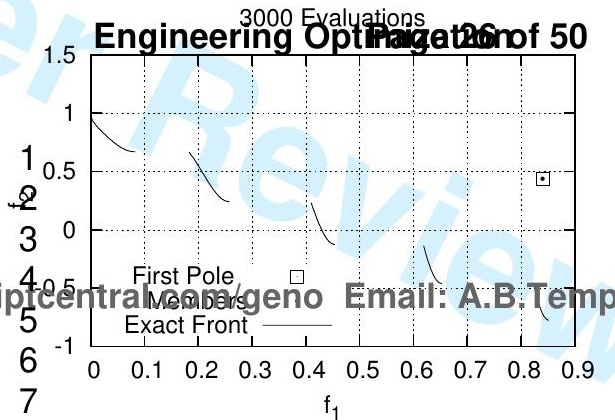




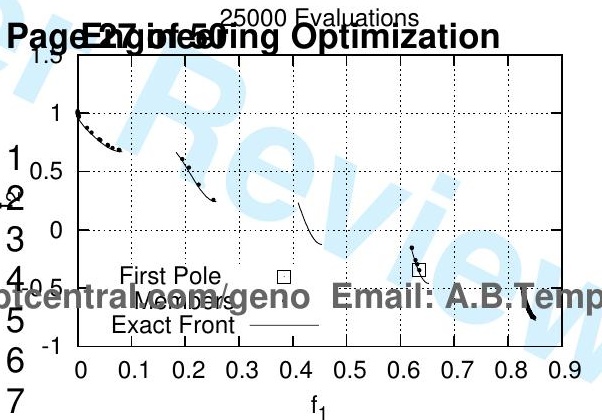




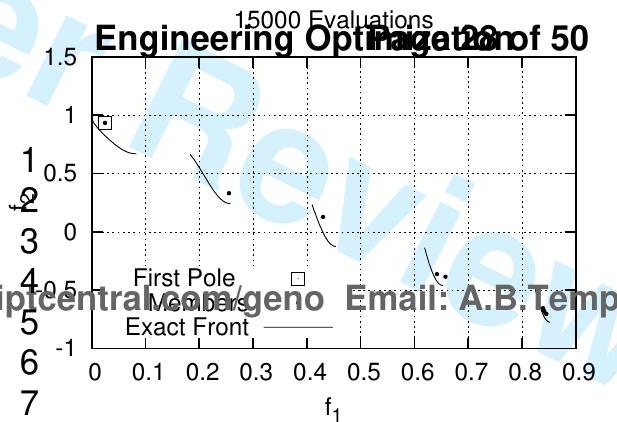




\subsection{5}

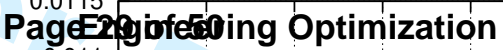
0.011

己े 0.01

$6 \times 6$ Mesh
$0.0105-\quad 8 \times 8$ Mesh $10 \times 10$ Mesh $12 \times 12$ Mesh

4 $6^{0.0085}$

$$
\begin{array}{lll}
0.34 & 0.36 & 0.38
\end{array}
$$

Émai B.Temp
$42 \quad 0.44$ 


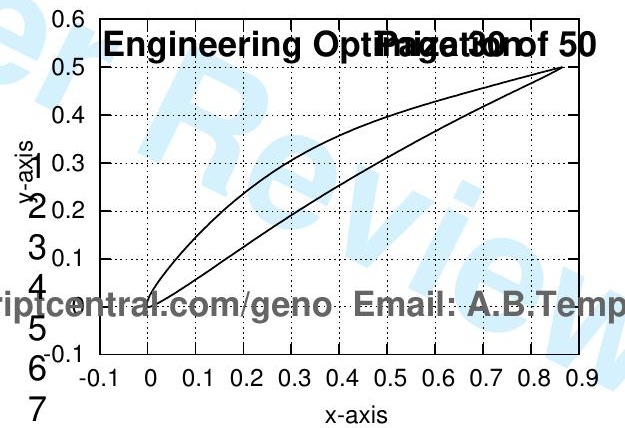




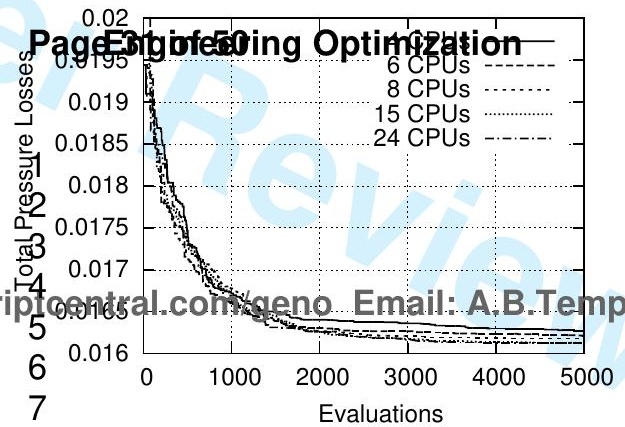




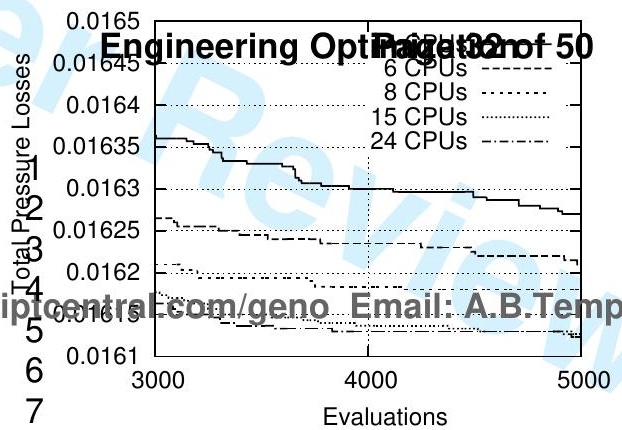




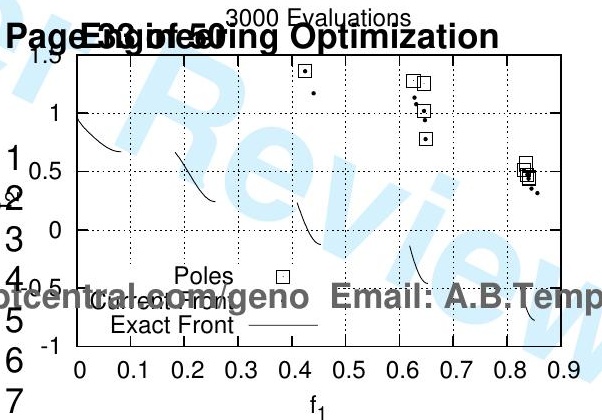




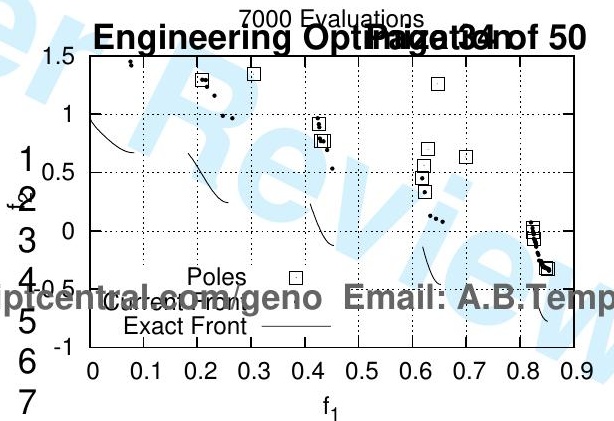




\section{Pag 10000 Evaluations}

PageEsojofeseoing Optimization

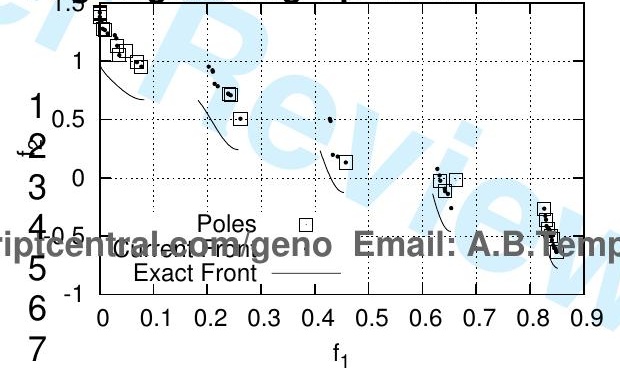




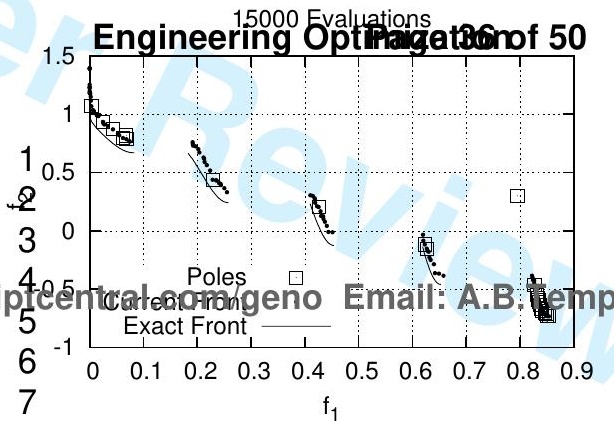




\section{Pagrion 20000 Evaluations}

PageEçgofeecing Optimization

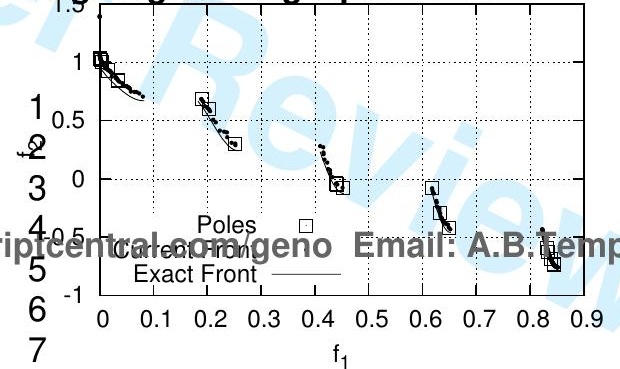




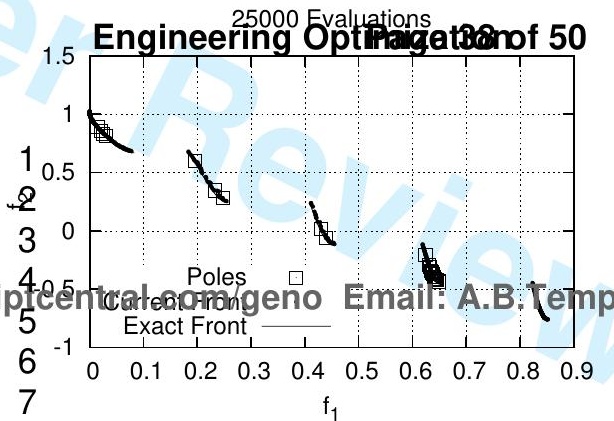


Page 39 of 50

Engineering Optimiz ion

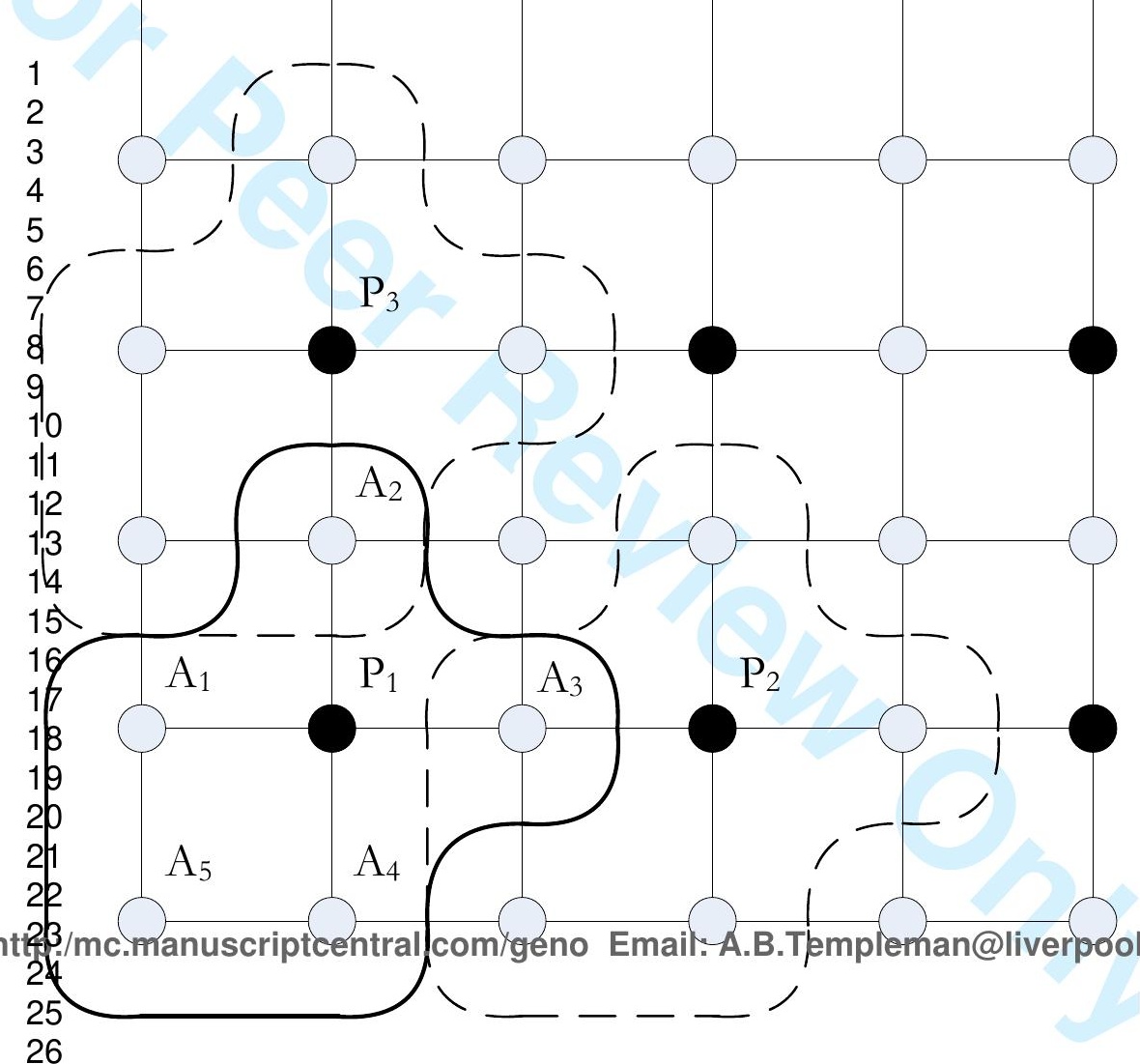




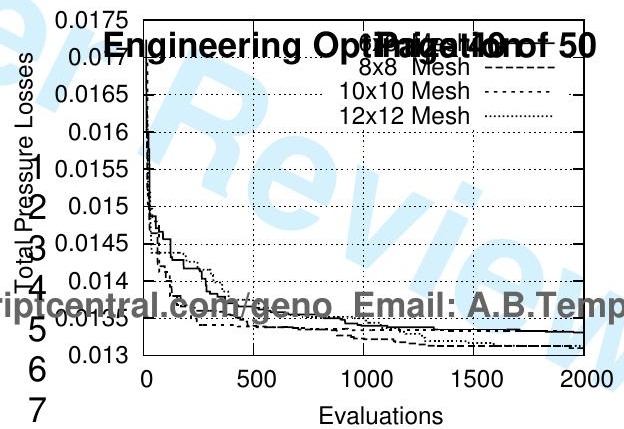




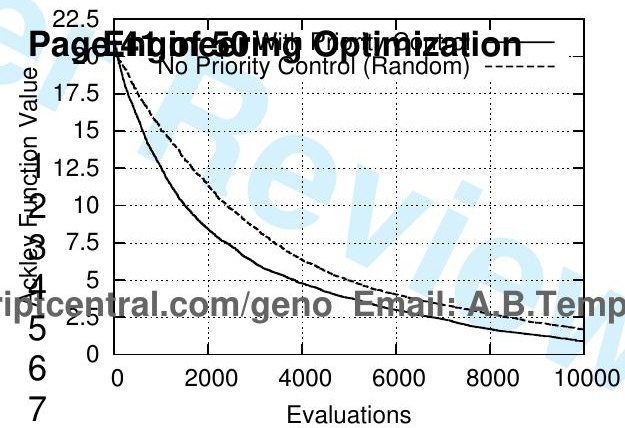




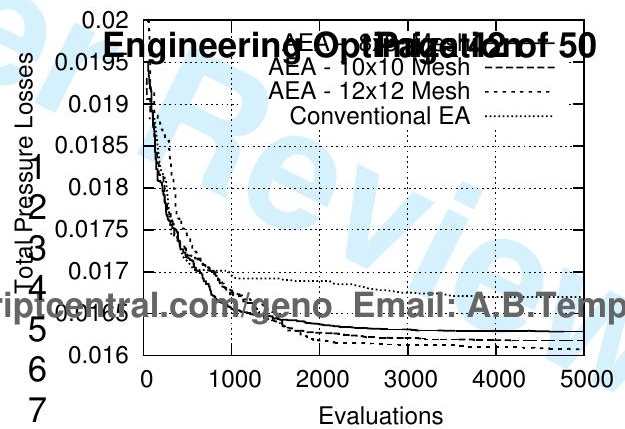




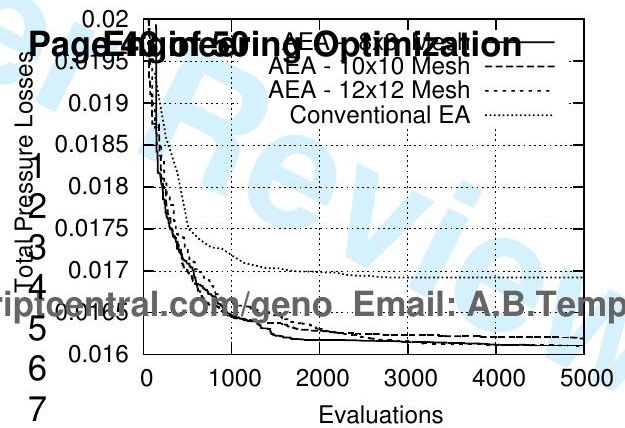




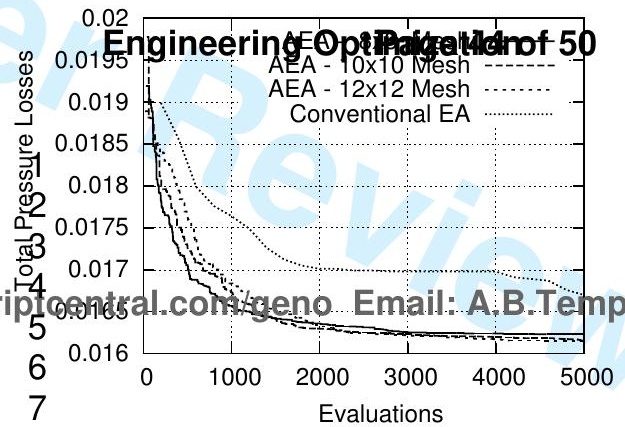




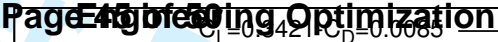 $C_{L}=0.3907-C_{D}=0.0095$ $\mathrm{C}_{\mathrm{L}}=0.4372-\mathrm{C}_{\mathrm{D}}=0.0112 \cdots \cdots$}

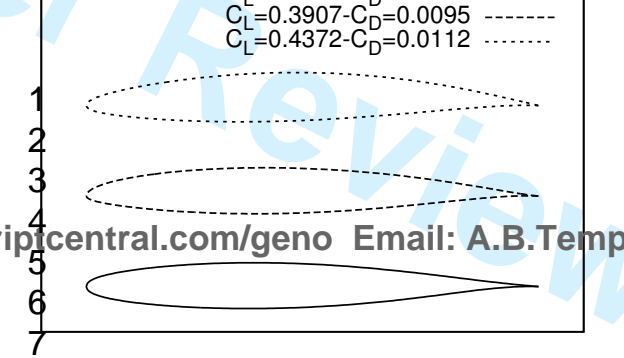




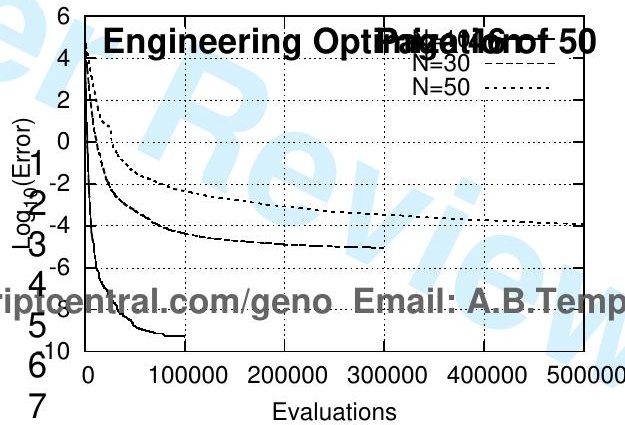




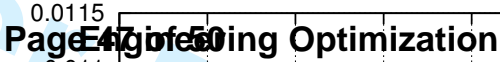
0.011

0.0105

己े 0.01 iptce.per

5

$6^{0.0085}$

$\begin{array}{lll}0.34 & 0.36 & 0.38\end{array}$

0.4

0.42

0.44

7

$\mathrm{C}_{\mathrm{L}}$ 


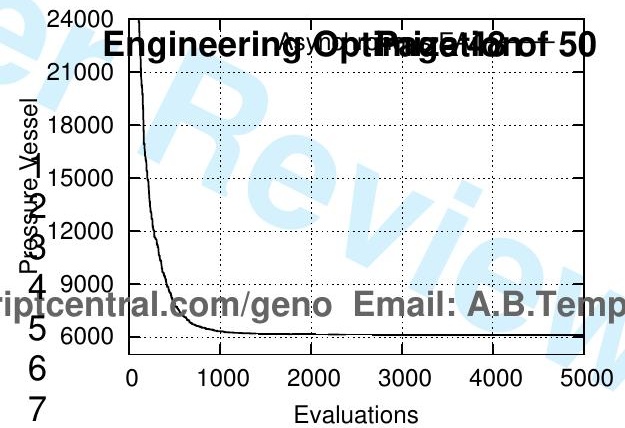




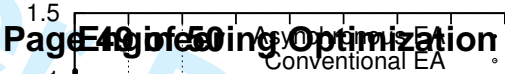

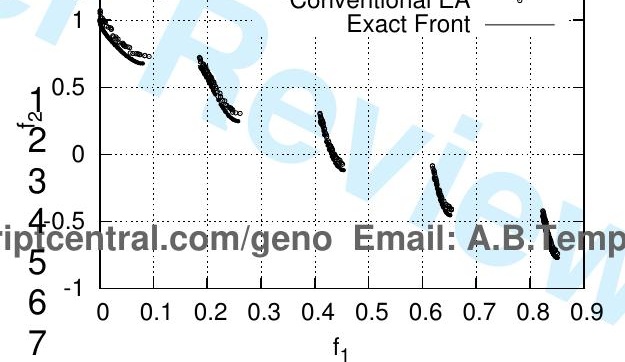




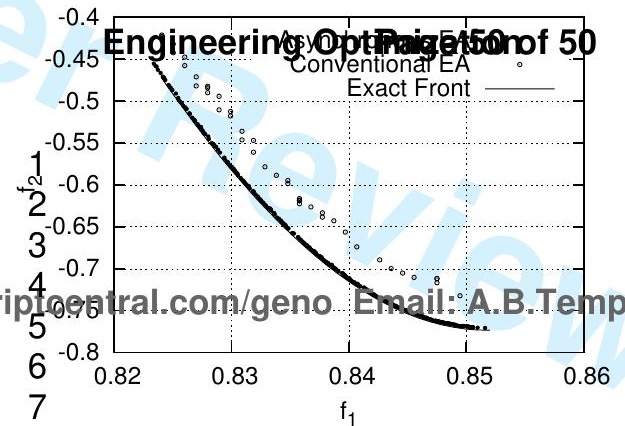

